INVITED ARTICLE

\title{
Partial differential equations for self-organization in cellular and developmental biology
}

\author{
Baker R E $\mathbf{E}^{1}$, Gaffney $\mathbf{E} \mathbf{A}^{1}$ and Maini $\mathbf{P} \mathbf{K}^{1,2}$ \\ ${ }^{1}$ Centre for Mathematical Biology, Mathematical Institute, University of Oxford, \\ 24-29 St Giles', Oxford OX1 3LB, UK \\ 2 Oxford Centre for Integrative Systems Biology, Department of Biochemistry, University \\ of Oxford, South Parks Road, Oxford OX1 3QU, UK \\ E-mail: baker@maths.ox.ac.uk,gaffney@maths.ox.ac.uk and maini@maths.ox.ac.uk
}

Received 19 June 2008, in final form 19 September 2008

Published 20 October 2008

Online at stacks.iop.org/Non/21/R251

Recommended by J A Glazier

\begin{abstract}
Understanding the mechanisms governing and regulating the emergence of structure and heterogeneity within cellular systems, such as the developing embryo, represents a multiscale challenge typifying current integrative biology research, namely, explaining the macroscale behaviour of a system from microscale dynamics. This review will focus upon modelling how cell-based dynamics orchestrate the emergence of higher level structure. After surveying representative biological examples and the models used to describe them, we will assess how developments at the scale of molecular biology have impacted on current theoretical frameworks, and the new modelling opportunities that are emerging as a result. We shall restrict our survey of mathematical approaches to partial differential equations and the tools required for their analysis. We will discuss the gap between the modelling abstraction and biological reality, the challenges this presents and highlight some open problems in the field.
\end{abstract}

Mathematics Subject Classification: 35-02; 35-R99; 92-02; 92B05; 92C15; 92C17; 92C37

PACS numbers: 82.40.Ck; 87.10.-e; 87.10.Ed; 87.17.Pq; 87.18.Ed; 87.18.Hf

(Some figures in this article are in colour only in the electronic version)

\section{Introduction}

The staggering variety in biological form is remarkable given the relatively small number of underlying mechanisms by which biological self-organization and morphogenesis proceed. In particular, the fundamentals governing how individual cell interactions create phenotypic 
structure are typically rooted in a relatively small number of similar principles, usually utilizing analogous, often homologous, proteins and evolutionary, conserved pathways [1]. It is differences in the temporal and spatial regulation of these mechanisms that typically result in the complexity that we see [1]. Consequently, our understanding of biological selforganization is often amenable to, and enhanced by, mathematical and computational modelling addressing the emergence and regulation of temporal and spatial complexity from fundamental mechanisms operating ultimately at a cellular level.

Given the importance of both spatial and temporal variation, partial differential equations (PDEs) are often, but certainly not always, a sensible mathematical framework for investigating large-scale structure formation. We will outline representative examples of such modelling investigations before reviewing how modern biological developments are revealing limitations in current modelling frameworks and highlighting exciting opportunities for novel theoretical studies.

\subsection{Examples of self-organization in cellular and developmental biology}

Developmental biology is replete with examples of self-organization. While the fertilized egg does exhibit polarity, it is a single cell from which numerous cell types, and structure, ultimately emerge. Questions immediately arise as to how do individual but genetically identical cells 'know' when and where to create or respond to signals, express genes, synthesize proteins, differentiate, migrate or divide. The principle mechanism for this is differential gene expression, whereby both long- and short-range signals are utilized to enforce tight spatial and temporal control over gene expression, thus robustly ensuring the pattern of differentiated cell types necessary for embryological development.

Numerous aspects of such dynamics have been considered from a theoretical perspective to systematically interrogate putative self-organization mechanisms, assist our insights and extract predictions of, or empirical tests for, their consequences. An illustrative selection of examples includes the following:

(a) mechanisms for axis formation, for example, left-right polarity initiation in the mouse [2-4];

(b) heterogeneous cell fate decisions, including the roles of Nodal and Lefty gene products in left-right polarity signal amplification [2,5], cell fates in the zebrafish mesoderm [6], the induction of neural tissue in the frog [1], patterning in the Drosophila wing [7] and hair follicle formation [8];

(c) controlled cell migrations, illustrated by gastrulation, lung morphogenesis and nervous system development [9-11];

(d) skeletal morphogenesis, for example the formation of somites [12, 13] and limbs [14-17];

(e) the formation of skin patterns, typified by mammalian coat markings [18-20] and feather bud arrangements [21, 22];

(f) fine-grained patterning, illustrated by lateral inhibition within delta-notch signalling [23];

(g) adhesion-driven cell sorting [24, 25].

Figure 1 illustrates four examples of self-organization in developmental biology, including coat markings, limb/digit, somite and feather bud formation. Cellular biological pattern formation is far from restricted to developmental biology, however. The formation and changes in the markings of fish, such as the Angelfish Pomacanthus [26] or the Foxn1-mutant mouse [27], are representative examples in which higher levels of marking structure evolve later than the developmental stage. In addition, there are numerous examples where the structure emerges because of interactions between individual organisms rather than within an 


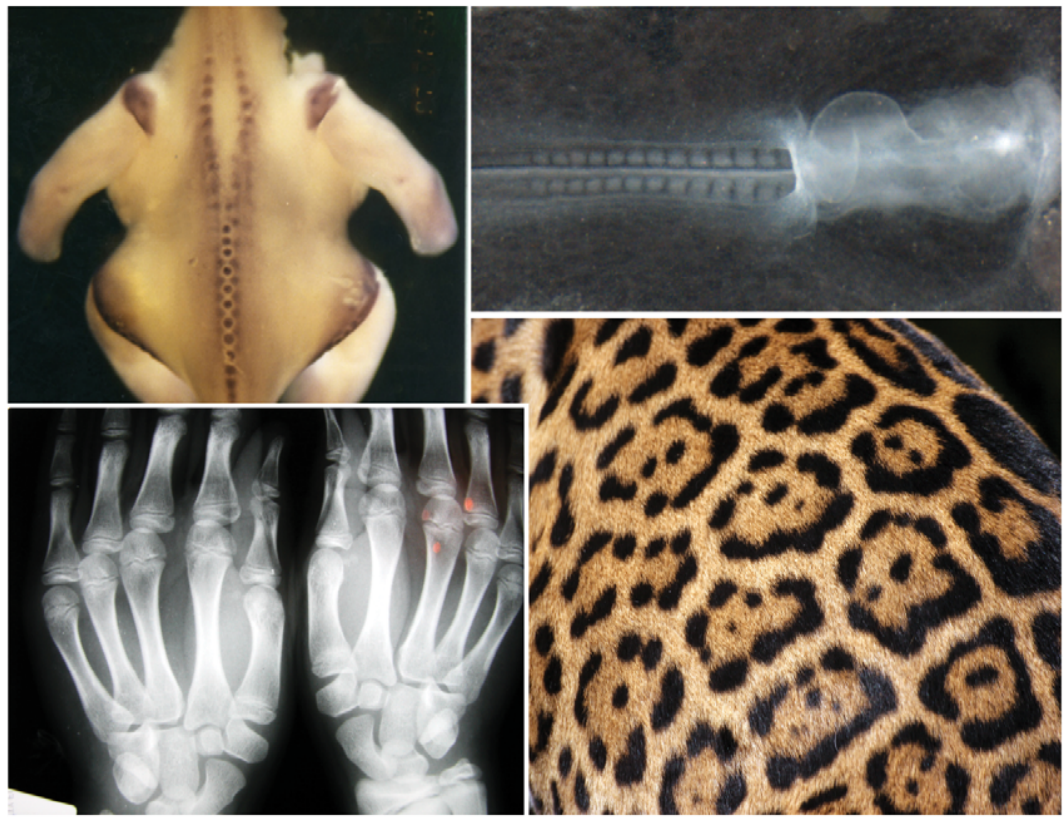

Figure 1. Examples of self-organization in biology. Clockwise from top-left: feather bud patterning, somite formation, jaguar coat markings, digit patterning. The first is reprinted from Widelitz et al 2000, $\beta$-catenin in epithelial morphogenesis: conversion of part of avian foot scales into feather buds with a mutated $\beta$-catenin Dev. Biol. 219 98-114, with permission from Elsevier. The second is courtesy of the Pourquié Laboratory, Stowers Institute for Medical Research, and the remainder are taken from the public image reference library at http://www.morguefile.com/.

(a) Activator

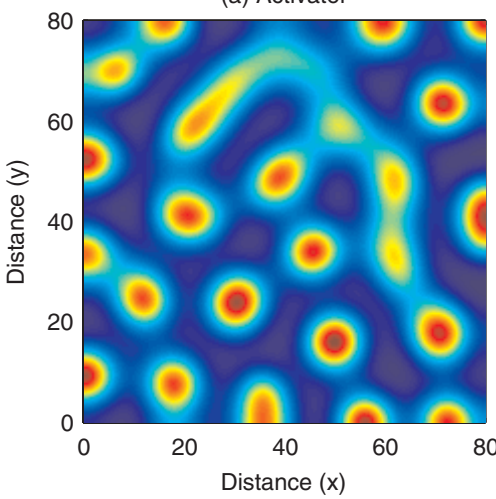

(b) Inhibitor

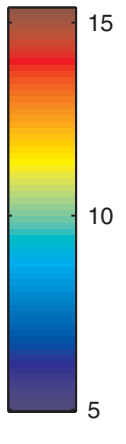

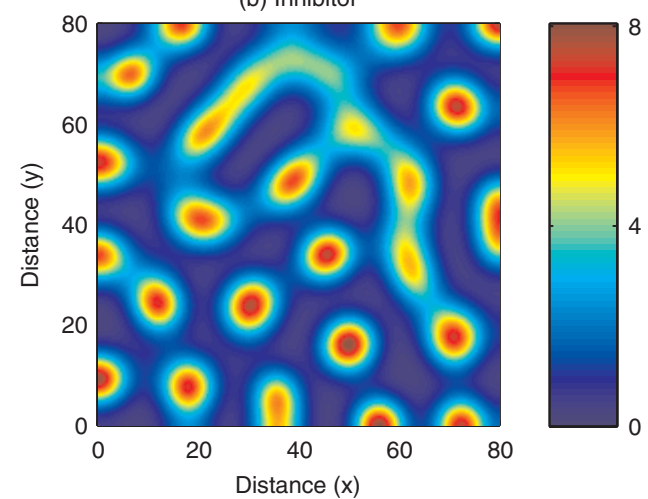

Figure 2. Numerical simulation of a Turing model with Gierer-Meinhardt kinetics, as given by equations (22) and (23). The field is initially at the homogeneous steady state, with small random perturbations added to the activator profile. Diffusion-driven amplification of the heterogeneities results in a steady state array of peaks and troughs; the activator $(u)$ and inhibitor $(v)$ are inphase'. Zero flux boundary conditions are imposed and $D=30, b=0.35$, as in [60]. The model equations were solved using a finite difference scheme, including complex step differentiation for the Jacobian evaluation [61]. 
individual organism. Common examples at the cellular level include self-organization due to the combination of motility and signalling within swarming bacteria $[28,29]$ and social amoebae such as Dictyostelium discoideum [30].

\subsection{A framework for PDE modelling of self-organization}

The most common principle for modelling self-organization in developmental biology using PDEs is the law of conservation [31]. With $\partial \Omega_{a}$ an arbitrary surface enclosing a timeindependent volume $\Omega_{a}$, then the rate of change in the amount of material inside $\Omega_{a}$ must equal the net flux of material across the surface $\partial \Omega_{a}$ plus the net production of material in $\Omega_{a}$. Thus

$$
\frac{\partial}{\partial t} \int_{\Omega_{a}} u(\boldsymbol{x}, t) \mathrm{d} V=-\int_{\partial \Omega_{a}} \boldsymbol{J} \cdot \mathrm{d} \boldsymbol{s}+\int_{\Omega_{a}} f(u, \boldsymbol{x}, t) \mathrm{d} V,
$$

where $u(\boldsymbol{x}, t)$ represents the amount of material at $\boldsymbol{x}$ at time $t, \boldsymbol{J}$ the flux of material and $f$ the net rate of production of $u$. Application of the divergence theorem yields

$$
\int_{\Omega_{a}}\left\{\frac{\partial u}{\partial t}+\nabla \cdot \boldsymbol{J}-f(u, \boldsymbol{x}, t)\right\} \mathrm{d} V=0 .
$$

Since the volume $\Omega_{a}$ is arbitrary, the integrand must be identically equal to zero and hence

$$
\frac{\partial u}{\partial t}=-\nabla \cdot \boldsymbol{J}+f(u, \boldsymbol{x}, t)
$$

The system is closed by specifying suitable initial and boundary conditions and the result can be generalized in an obvious manner to include multiple, interacting species.

1.2.1. Derivation of the flux. The material flux can arise from many sources and its specification becomes the next problem to be faced. Most simple is advection of the material with velocity $\boldsymbol{v}$; this results in a flux of the form $\boldsymbol{J}=\boldsymbol{v} u$. Diffusion is an alternative, common, transport mechanism; most frequently employed is the Fickian formulation, $\boldsymbol{J}=-D \nabla u$, which yields

$$
\frac{\partial u}{\partial t}=D \nabla^{2} u+f(u, \boldsymbol{x}, t)
$$

so that the material moves down gradients of its own density. However, such a formulation tends to imply that diffusion is a short-range effect: this can be seen by considering the Laplacian operator in the absence of material production [31]:

$$
\nabla^{2} u \propto \lim _{R \rightarrow 0} \frac{\langle u(\boldsymbol{x}, t)\rangle-u(\boldsymbol{x}, t)}{R^{2}},
$$

where $\langle u(\boldsymbol{x}, t)\rangle$ is the average density of material in a sphere of radius $R$ centred about the point $\boldsymbol{x}$,

$$
\langle u(\boldsymbol{x}, t)\rangle=\frac{3}{4 \pi R^{3}} \int_{\text {sphere }} u(\boldsymbol{x}+\boldsymbol{r}, t) \mathrm{d} \boldsymbol{r} .
$$

Expansion of the integrand about $\boldsymbol{x}$ for small $\boldsymbol{r}$ gives

$$
\begin{aligned}
\langle u(\boldsymbol{x}, t)\rangle & =\frac{3}{4 \pi R^{3}} \int_{\text {sphere }}\left[u(\boldsymbol{x}, t)+(\boldsymbol{r} . \nabla) u(\boldsymbol{x}, t)+\frac{1}{2}(\boldsymbol{r} . \nabla)^{2} u(\boldsymbol{x}, t)+\cdots\right] \mathrm{d} \boldsymbol{r}, \\
& =u(\boldsymbol{x}, t)+\frac{1}{10} R^{2} \nabla^{2} u(\boldsymbol{x}, t)+\cdots,
\end{aligned}
$$

since the second term in the integrand evaluates to zero. This is of the form of equation (5) with constant of proportionality $1 / 10$.

Other forms for the flux are discussed in more detail in section 2.2-in general they assume phenomenological forms which are based on qualitative observations. 
1.2.2. Integro-differential formulation. Another common framework is an integral formulation, so that the rate of change in material density at $\boldsymbol{x}$ is dependent on the density at neighbouring positions $\boldsymbol{x}+\boldsymbol{r}$. This leads to an integro-differential equation of the form

$$
\frac{\partial u}{\partial t}=f(u, \boldsymbol{x}, t)+\int_{\mathbb{R}^{3}} w(\boldsymbol{r}) u(\boldsymbol{x}+\boldsymbol{r}, t) \mathrm{d} \boldsymbol{r},
$$

where $w$ is a kernel which quantifies the effect of material in the environment upon $u(\boldsymbol{x}, t)$. Any constraints placed on $w$ are dependent on the biological system under consideration. Typically one might assume that $w$ is homogeneous and isotropic and that the influence becomes negligible as the distance from $\boldsymbol{x}$ becomes large [32]:

$$
w \rightarrow 0 \quad \text { as } \quad|\boldsymbol{r}| \rightarrow \infty, \quad w=w(|\boldsymbol{r}|) .
$$

Such formulations have been used to model, for example, the effects of cell adhesion and cell sorting according to different cell-cell adhesion strengths [24,33].

A Taylor expansion of the density function contained in the integrand, on use of the isotropy of $w$, gives rise to

$$
\begin{aligned}
\frac{\partial u}{\partial t} & =f(u, \boldsymbol{x}, t)+\int_{\mathbb{R}^{3}} w(\boldsymbol{r})\left[u(\boldsymbol{x}, t)+(\boldsymbol{r} \cdot \nabla) u(\boldsymbol{x}, t)+\frac{1}{2}(\boldsymbol{r} \cdot \nabla)^{2} u(\boldsymbol{x}, t)+\cdots\right] \mathrm{d} \boldsymbol{r}, \\
& =f(u, \boldsymbol{x}, t)+w_{0} u+w_{2} \nabla \cdot(\nabla u)+w_{4} \nabla \cdot \nabla\left(\nabla^{2} u\right)+\cdots,
\end{aligned}
$$

where, assuming convergence,

$$
w_{2 j}=\frac{1}{(2 j) !} \int_{\mathbb{R}^{3}} \boldsymbol{r}^{2 j} w(\boldsymbol{r}) \mathrm{d} \boldsymbol{r} .
$$

Depending on the form of $w$, it may be expedient to truncate the expansion after a finite number of terms, yielding a higher order PDE. For example, it is immediately obvious that truncation of the approximation after the 4 th order contribution results in an equation of the form

$$
\frac{\partial u}{\partial t}=f(u, \boldsymbol{x}, t)+\nabla \cdot\left[D_{1} \nabla u-D_{2} \nabla\left(\nabla^{2} u\right)\right] .
$$

This type of formulation is often used by modellers to describe the ability of cells to sense non-locally due to their finite size—see, for example, [34].

1.2.3. Alternative derivations. There are, of course, several other methods of deriving PDE descriptions of movement-we briefly outline a couple in this section.

Velocity jump processes, in 1D for example, consider the density of left- and rightmoving particles which reverse their direction of motion at random instants of time at rate $\lambda$, say. Denoting the probability densities of left- and right-moving individuals by $p_{-}$and $p_{+}$, respectively, and particle speed by $s>0$ gives [35]

$$
\begin{aligned}
& \frac{\partial p_{-}}{\partial t}-s \frac{\partial p_{-}}{\partial x}=\lambda\left(p_{+}-p_{-}\right), \\
& \frac{\partial p_{+}}{\partial t}+s \frac{\partial p_{+}}{\partial x}=\lambda\left(p_{-}-p_{+}\right) .
\end{aligned}
$$

Letting $p=p_{-}+p_{+}$gives the Telegraph equation [36]

$$
\frac{\partial^{2} p}{\partial t^{2}}+2 \lambda \frac{\partial p}{\partial t}=s^{2} \frac{\partial^{2} p}{\partial x^{2}}
$$

which in the limit $\lambda \rightarrow \infty$ and $\lim _{\lambda \rightarrow \infty} s^{2} / 2 \lambda \rightarrow D$ (constant) results in the classical diffusion equation, excluding behaviour at the extremes of the wavefront where the singular asymptotic limit, switching between hyperbolic and parabolic behaviour, becomes important. 
On the other hand, space jump processes suppose particles change their position at random instants of time. Considering jumps of length $h$, space can be denoted by a set of grid points, $x_{i}$, of separation $h$, such that $P_{i}(t)$, the probability of finding an individual at $x_{i}$ at time $t$, evolves according to the equation [37]

$$
\frac{\partial P_{i}}{\partial t}=d\left[P_{i-1}(t)-2 P_{i}(t)+P_{i+1}(t)\right]
$$

where $d$ is the (constant) transition rate per unit time. Letting the step size, $h$, tend to zero, expanding the terms $P_{i \pm 1}$ about $x_{i}$ and assuming that $\lim _{h \rightarrow 0} d h^{2}=D$ gives once again the diffusion equation.

These formulations, and others, have been derived in considerably more generality than we can feasibly survey here and we refer interested readers to the literature [35, 37-39].

\subsection{The suitability of PDEs for modelling biological self-organization}

As motivated above, classical modelling studies in biological self-organization have often focused on differential equations. For simple systems, exact analytical solutions may be possible. Where they are not, separation of space and time scales or the exploitation of some other small parameter enables the use of multiscale asymptotic approaches which give excellent insight into system behaviour under different parameter regimes. As the number of model components becomes too unwieldy or the interactions too complex for such approaches, increasingly sophisticated computational methods allow accurate numerical approximations to be calculated over a wide range of parameter space.

Nonetheless, one should be aware of the limitations of this type of modelling. The flux and/or production terms in the conservation formulation are often phenomenological, without derivation from universal or fundamental principles. In addition, as the material density becomes low stochastic effects can become significant. Finally, tortuous cellular level geometry complicates the investigation of spatial fluctuations at the cellular scale and the possibility of large variations among neighbouring cells prevents straightforward use of a continuum limit.

However, with careful consideration of these pitfalls and awareness of when and where techniques can successfully be applied, PDEs remain one of the most useful and insightful tools for modelling self-organization in developmental biology.

\subsection{Wider context}

Although this review will focus on self-organization in biology, it is important to note that the general phenomenon of pattern formation has motivated an enormous amount of mathematical modelling and analysis, most of which is beyond the scope of this review. However, we refer interested readers to the seminal paper of Cross and Hohenberg [40], which reviews theoretical approaches to spatial pattern formation in hydrodynamics, solidification fronts, nonlinear optics and chemical reactions, in addition to biological phenomena, and the books by Britton [41], Fife [42], Grindrod [43] and Hoyle [44]. These references carry out detailed analytic bifurcation studies of coupled systems of PDEs using a wide range of mathematical approaches to address issues such as pattern selection, marginal stability, effects of boundaries and geometry, as well as investigating oscillatory behaviour, propagation of pulses and fronts, external forcing, effects of external noise, chaos and turbulence. 


\section{An overview of the PDE models of self-organization}

It is possible to classify the archetypal PDE models for self-organization in development into two broad categories. In the first, chemicals (termed morphogens) specify a pre-pattern for future differentiation without interaction from the cells themselves. In the second, patterning occurs with feedback from the cells on any chemical or physical constituents that may be playing a role in the organization of the system; these are generally referred to as taxis models. Both can be derived from conservation equations taking the form

$$
\begin{aligned}
& \frac{\partial \boldsymbol{n}}{\partial t}=-\nabla \cdot \boldsymbol{J}^{n}+\boldsymbol{f}^{n}(\boldsymbol{n}, \boldsymbol{u}, \boldsymbol{x}, t), \\
& \frac{\partial \boldsymbol{u}}{\partial t}=-\nabla \cdot \boldsymbol{J}^{u}+\boldsymbol{f}^{u}(\boldsymbol{n}, \boldsymbol{u}, \boldsymbol{x}, t),
\end{aligned}
$$

where $\boldsymbol{u}$ represents the material concentration of biochemical species and $\boldsymbol{n}$ the density of different cell types. $\boldsymbol{J}^{n / u}$ and $\boldsymbol{f}^{n / u}$ are as before but, importantly, they now also describe the interactions between the model species.

In order to distinguish between pre-pattern and taxis models, we non-dimensionalize in the following manner:

$$
\begin{aligned}
& \frac{\partial \tilde{\boldsymbol{n}}}{\partial \tilde{t}}=-\nabla \cdot \tilde{\boldsymbol{J}}^{n}+\tilde{\boldsymbol{f}}^{n}(\tilde{\boldsymbol{n}}, \tilde{\boldsymbol{u}}, \tilde{\boldsymbol{x}}, \tilde{t}), \\
& \eta \frac{\partial \tilde{\boldsymbol{u}}}{\partial \tilde{t}}=-\nabla \cdot \tilde{\boldsymbol{J}}^{u}+\tilde{\boldsymbol{f}}^{u}(\tilde{\boldsymbol{n}}, \tilde{\boldsymbol{u}}, \tilde{\boldsymbol{x}}, \tilde{t}),
\end{aligned}
$$

where the tildes denote non-dimensional variables and $\eta$ is the ratio of time scales of biochemical changes to the cellular response (to biochemical signals). A number of possible cases arise, two of which are most interesting from the point of view of discussions here. Firstly, in the case that $\boldsymbol{J}^{u}$ and $\boldsymbol{f}^{u}$ are independent of $\boldsymbol{n}$, the chemical dynamics decouple from the system and cellular dynamics are slave to the chemical pattern. Further, when $\eta \ll 1$, so that the cellular response occurs significantly more slowly than the time scale of biochemical changes, the biochemical species are at a steady state to leading order in $\eta$, at least once any initial transients have dissipated. Consequently, we have a pre-pattern that directs future cell differentiation.

In the rest of this section we give an overview of the paradigm models for self-organization, outlining in each case examples of their use in modelling developmental systems.

\subsection{Morphogen models}

A morphogen refers to a diffusible signalling molecule, typically within a developmental setting, that exerts a graded effect. In particular, cells exhibit a differential response to the morphogen concentration $[1,45]$ or possibly the history of morphogen concentration $[46,47]$. A standard example is the response of cells surrounding a source of morphogen, whereby the gradient in morphogen concentration induces a spatially heterogeneous cellular response. Textbook examples of this include the Sonic hedgehog (Shh) protein in chick limb development [48-50] and the signalling protein Decapentaplegic (Dpp) in Drosophila wing imaginal disc development [51, 52]. Often, the long-range action of a morphogen is curtailed by the effects of an antagonist, or inhibitor, and it is the interplay of these proteins that determines the ultimate response; examples include the induction of neural tissue in the frog [1] and specification of the somites $[53,54]$. Generally this conceptual framework is standard in development $[1,45]$. A modelling framework for such systems via reaction-diffusion PDEs is

$$
\frac{\partial \boldsymbol{u}}{\partial t}=\nabla \cdot(\boldsymbol{D} \nabla \boldsymbol{u})+\boldsymbol{f}(\boldsymbol{u}, \boldsymbol{x}, t),
$$


on a bounded domain, usually with Neumann, Dirichlet or Robin boundary conditions. Sources and sinks of morphogens can be incorporated via Neumann conditions and/or included in the function $f$. In complete generality one may consider such equations on numerous neighbouring domains or with a spatially varying diffusion matrix, $\boldsymbol{D}$. An example of such a model considers the robustness of patterning due to the gradient of the signalling molecule BMP in the dorsal region of the Drosophila embryo where the domain is split into three regions (the neural ectoderm, the mesoderm and the dorsal region) [55].

2.1.1. Turing models. An important special case of the morphogen model, ubiquitous in theoretical studies, is the eponymous Turing model first considered in the 1950s [56]. This imposes additional constraints on $\boldsymbol{D}$ and $\boldsymbol{f}$ in equation (21) and precludes the possibility of spatially inhomogeneous transport or reaction coefficients. In particular, in the absence of diffusion, and thus spatial heterogeneity, the model exhibits a stable homogeneous steady state. This steady state is however also required to be unstable in the presence of diffusion. The fact that stability is lost on introducing spatial heterogeneity via a diffusive transport process is initially counter-intuitive in that diffusion, in the absence of reaction kinetics, acts to drive a system to its homogeneous steady state, rather than destabilize it. General, necessary and sufficient, conditions for a Turing instability for equations (21) on an $n$-dimensional spatial domain are presented in the literature [57] and two component analysis can be found in most textbooks (see below and, e.g. [32]).

In particular, for a system consisting of two morphogens one requires the concept of 'short-range activation, long-range inhibition' for a Turing model [58]. Thus one of the morphogens, denoted the activator, has to catalyze its own production and be inhibited by the second morphogen. In addition, the activator has to have a smaller diffusion coefficient and thus have a smaller range of influence. Without these biologically restrictive constraints, a Turing instability cannot occur.

Figure 2 shows the results of the numerical simulation of a Turing morphogen model using Gierer-Meinhardt kinetics [59] of the form

$$
\begin{aligned}
& \frac{\partial u}{\partial t}=\nabla \cdot(\nabla u)+\frac{u^{2}}{v}-b u, \\
& \frac{\partial v}{\partial t}=\nabla \cdot(D \nabla v)+u^{2}-v,
\end{aligned}
$$

where $D$ and $b$ are positive constants. Amplification of small fluctuations in the activator field leads to a regular array of peaks and troughs in both activator and inhibitor concentrations.

An important consequence of such a model is that for a sufficiently small domain, significantly smaller than the diffusive length scale, the homogeneous steady state will be stable. However, increasing the domain size results in the diffusively driven spatial variation becoming non-negligible and thus a bifurcation to spatial heterogeneity. This provides a mechanism for the initiation of structure from fluctuations. As such, this subset of morphogen models is more fundamental than the general model. When examining the consequence of distributions of BMP in models such as those presented by Eldar [55], one considers the downstream effects of an implicit earlier heterogeneity (for example, the fact that the domain is already split into three regions). In contrast, Turing models are capable of representing the initial symmetry breaking event and/or amplifying the smallest of fluctuations into a large-scale spatial pattern.

This is a very important distinction. Morphogen-based pattern formation, whereby signalling molecules' long-range influence induces a higher order of structure, is a common and validated paradigm in developmental biology $[1,45]$. The Turing pattern formation mechanism 
within a morphogen system, in contrast, is still to be definitively validated in developmental biology. Potential candidates for Turing morphogens are Nodal and Lefty in the amplification of an initial signal of left-right axis formation [2,5] and zebrafish mesoderm cell fates [6], plus Wnt and $D k k$ in hair follicle formation [8] and TGF- $\beta$ as the activator, plus an unknown inhibitor, in limb bud morphogenesis [62].

A difficulty with the Turing model concerns the ratio of activator to inhibitor diffusion coefficients. Even models which consider a direct comparison with experiments take this ratio to be much less than unity; for example, in [8] it is taken to be 0.025 without biological motivation. However, it is unlikely that such small ratios exist in the absence of a large ratio of molecular sizes for the Turing morphogens. Arguably unrealistic ratios of diffusion coefficients for ATP and ADP have also been used in simple models illustrating that Turing patterns can potentially occur at the subcellular level for the glycolytic reaction [63]. In preparation for our discussion on future modelling opportunities, we will show that while Turing patterns can occur in two variable systems with nearly equal diffusion rates, this requires an extreme fine-tuning of reaction rate constants.

The Turing instability for nearly equal diffusion coefficients. Consider a two component system $\boldsymbol{u}(\boldsymbol{x}, t)=(u(\boldsymbol{x}, t), v(\boldsymbol{x}, t))$ satisfying the reaction-diffusion equations

$$
\frac{\partial \boldsymbol{u}}{\partial t}=\boldsymbol{D} \nabla^{2} \boldsymbol{u}+\boldsymbol{f}(\boldsymbol{u}), \quad \boldsymbol{x} \in \Omega, \quad t \in[0, \infty),
$$

where $\boldsymbol{D}=\operatorname{diag}\left(D_{1}, D_{2}\right)$ is a diffusion matrix, $\boldsymbol{f}(\boldsymbol{u})=(f(u, v), g(u, v)), \Omega$ is a bounded domain and the initial conditions are denoted by $\boldsymbol{u}(\boldsymbol{x}, 0)=\boldsymbol{u}_{0}(\boldsymbol{x})$. For definiteness, we consider homogeneous Neumann boundary conditions, that is

$$
\boldsymbol{n} \cdot \nabla u=\boldsymbol{n} \cdot \nabla v=0, \quad \boldsymbol{x} \in \partial \Omega,
$$

where $\boldsymbol{n}$ is the outward pointing normal on $\partial \Omega$. We assume the kinetics are such that there is a unique, positive, homogeneous, linearly stable steady state, denoted $\boldsymbol{u}_{*}$. Now consider a small perturbation about this steady state of the form

$$
\boldsymbol{u}(\boldsymbol{x}, t)=\boldsymbol{u}_{*}+\epsilon \operatorname{Re}\left[\mathrm{e}^{\lambda_{k} t} \boldsymbol{u}_{k} w_{k}(\boldsymbol{x})\right],
$$

where $\boldsymbol{u}_{k}$ is a constant vector and the function $w_{k}(\boldsymbol{x})$ is not identically zero and satisfies the eigenfunction equation

$$
\nabla^{2} w_{k}=-k^{2} w_{k}
$$

on the domain $\Omega$, inheriting boundary conditions from the full problem. This fixes the allowed, constant, values of $k$.

Note that $k$ must be real. Indeed, suppose $\nabla^{2} w=\mu w$, where $\mu$ and $w$ are, in general, complex and $\mu$ is constant. Then a standard manipulation of equation (27) and its complex conjugate firstly yields

$$
\left(\mu^{*}-\mu\right) \int_{\Omega}|w|^{2} \mathrm{~d} V=\int_{\Omega}\left[w \nabla^{2} w^{*}-w^{*} \nabla^{2} w\right] \mathrm{d} V=0,
$$

and thus the eigenvalue, $\mu$, must be real. Its negativity follows from

$$
0=\int_{\Omega}\left[w^{*} \nabla^{2} w-\mu w^{*} w\right] \mathrm{d} V
$$

which implies

$$
\mu=-\frac{\int_{\Omega} \nabla w \cdot \nabla w^{*} \mathrm{~d} V}{\int_{\Omega} w^{*} w \mathrm{~d} V}=-\frac{\int_{\Omega}|\nabla w|^{2} \mathrm{~d} V}{\int_{\Omega}|w|^{2} \mathrm{~d} V} \leqslant 0 .
$$


Thus we can write $\mu=-k^{2}$, with $k$ real.

We proceed by substituting (26) into (24) and working to leading orders in $\epsilon$, as befits a linear stability analysis. This reveals that a non-trivial perturbation requires

$$
\operatorname{det}\left|\lambda_{k} \boldsymbol{I}-\boldsymbol{J}+k^{2} \boldsymbol{D}\right|=0,
$$

where

$$
\boldsymbol{J}=\left(\begin{array}{ll}
f_{u} & f_{v} \\
g_{u} & g_{v}
\end{array}\right)
$$

is the Jacobian matrix of the kinetics $\boldsymbol{f}$ evaluated at the steady state $\boldsymbol{u}_{*}$. A Turing instability, i.e. a diffusively driven instability, requires both (i) $\operatorname{Re}\left(\lambda_{0}\right)<0$ and (ii) $\operatorname{Re}\left(\lambda_{k}\right)>0$ for some allowed value of $k \neq 0$. Condition (i) yields

$$
f_{u} g_{v}-f_{v} g_{u}>0, \quad f_{u}+g_{v}<0 .
$$

Condition (ii) additionally reveals that we require

$$
D_{1} D_{2} k^{4}-\left(D_{2} f_{u}+D_{1} g_{v}\right) k^{2}+\left(f_{u} g_{v}-g_{u} f_{v}\right)<0,
$$

and hence that

$$
D_{2} f_{u}+D_{1} g_{v}>0, \quad\left(D_{2} f_{u}+D_{1} g_{v}\right)^{2}>4 D_{1} D_{2}\left(f_{u} g_{v}-g_{u} f_{v}\right),
$$

for an allowed value of $k$. For almost equal diffusion coefficients we can write $D_{2}=D_{1}(1+\delta)$ with $|\delta| \ll 1$. Without loss of generality we take $\delta>0$, which forces $f_{u}>0$. Then one finds that the existence of a Turing instability requires that the constraint

$$
-\frac{g_{v}}{f_{u}} \in(1,1+\delta),
$$

is satisfied. This entails we can immediately write $g_{v}=-f_{u}\left(1+\chi_{1} \delta\right)$ for some $\chi_{1} \in(0,1)$. Reconsidering the second inequality in each of (33) and (35) now reveals

$$
1+\frac{\delta^{2}}{4} \frac{\left(1-\chi_{1}\right)^{2}}{\left[1+\chi_{1} \delta\right][1+\delta]}>\frac{g_{u} f_{v}}{f_{u} g_{v}}>1,
$$

which is an extremely fine-tuned constraint to impose on the kinetics. For example, a relative difference of $25 \%$ in the diffusion coefficients, corresponding to $\delta=1 / 4$, requires a fine-tuning in the kinetic functions at levels which are bounded above by

$$
\frac{\delta^{2}}{4[1+\delta]},
$$

which is $1 / 80$, equivalent to $1.25 \%$. Note that this is a bound whose attainment requires an additional fine-tuning of $\chi_{1}$; taking a value of $\chi_{1}$ which is intermediate in its allowed range, for definiteness $1 / 2$, will require a greater fine-tuning, at the $0.3 \%$ level, for a Turing instability.

However, a fine-tuning of reaction rates in the kinetics is difficult to explain in biology as they are subject to abundant fluctuation [64]. Given numerous biological systems do appear to have at least a Turing-type behaviour; this is perhaps a paradox and thus also a challenge and opportunity for further modelling work to which we will return below.

\subsection{Cell movement and taxis models}

As mentioned previously, an implicit assumption of the morphogen models is that the cellular response to the signal inherent in the distribution of morphogens occurs before, or on a much faster time scale than, cell migration and growth. This assumption consequently decouples the morphogen model from cell migration or growth, allowing it to be considered in isolation. It is obvious, however, that this assumption need not always be true, giving rise to a more complex 
modelling framework, where the cellular response to a signal cannot be de-coupled from cell dynamics.

Taxis, in a biological context, is the preferential movement of cells towards or away from a cue. Frequently the cue is a biochemical gradient which in a developmental context can be considered to be a morphogen, though more generally the chemical cue can be a nutrient or a metabolite, for example, or the cue can be non-chemical. There are numerous forms of taxis that can be observed; a non-exhaustive list includes where the cue is due to cellular adhesion sites (haptotaxis) [65-67], substrate stiffness (mechanotaxis) [68-70], light (phototaxis) [71], an electric potential (galvanotaxis) [72], a magnetic field (magnetotaxis) [73], the direction of gravity (geotaxis) [71, 74] and the balance of viscous and gravitational torques (gyrotaxis) [75]. For brevity, we will focus specifically on models which are relevant for development and/or the cue is chemotactic_-other forms may be treated in a similar fashion.

2.2.1. Chemotaxis models. Chemotaxis has been implicated in many developmental settings, especially in inducing and directing cell motility, leading to higher level structure on the developing embryo. Examples include the formation of the gut (gastrulation) [10,11], lung morphogenesis [9] and feather bud formation [22]. In addition to modelling chemotaxis in development [76,77], such models are also commonly considered for coat marking patterns [78] and swarming microbe motility [28-30].

To model chemotaxis we need equations representing the density of each motile cell type. We also need equations representing the signalling molecules, which are referred to as chemoattractants or chemorepellents according to how they influence cell motility. Then we have, in many instances,

$$
\begin{aligned}
& \boldsymbol{n}_{t}=\nabla \cdot\left(\boldsymbol{D}_{n} \nabla n\right)-\nabla \cdot \boldsymbol{J}^{c}+\boldsymbol{f}(\boldsymbol{n}, \boldsymbol{u}), \\
& \boldsymbol{u}_{t}=\nabla \cdot\left(\boldsymbol{D}_{u} \nabla u\right)+\boldsymbol{g}(\boldsymbol{n}, \boldsymbol{u}),
\end{aligned}
$$

where $\boldsymbol{n}$ and $\boldsymbol{u}$ are vectors describing cell density and chemical concentration, respectively. $\boldsymbol{D}_{n}$ and $\boldsymbol{D}_{u}$ are diffusivity matrices, as described above for the morphogen models, though typically $\boldsymbol{D}_{n}$ is positive definite rather than positive semi-definite. The functions $\boldsymbol{f}(\boldsymbol{n}, \boldsymbol{u})$ and $\boldsymbol{g}(\boldsymbol{n}, \boldsymbol{u})$ capture signalling molecule interactions, plus typically their production and/or degradation by the cells, plus other processes such as cell proliferation and death. The term $\boldsymbol{J}^{c}$ describes the flux of cells due to chemotaxis. Given that the cell response is directional it is, in the simplest setting, taken to be contingent on the gradient of the chemoattractants and chemorepellents. This yields for the $k$ th cell type

$$
J_{k}^{c}=n_{k} \sum_{i} \chi_{k, i}\left(u_{i}\right) \nabla u_{i}
$$

so that $\chi_{k, i}$, the chemotactic sensitivity, dictates the response of the $k$ th cell type to the $i$ th chemical. Note that if $\chi_{k, i}<0$, the flux is down concentration gradients and hence the cell type $k$ is repelled by chemical $i$. The typical forms of $\chi_{k, i}\left(u_{i}\right)$ are

$$
\chi_{k, i}\left(u_{i}\right)=\frac{\chi_{0}}{\left(K+u_{i}\right)^{j}},
$$

where $K>0, \chi_{0}$ are constants with $j \in\{0,1,2\}$. These functional forms emerge from receptor models $[79,80]$ though the specific choices of $\chi_{0}, K, j$ are cell- and context-dependent.

Figure 3 shows the results of numerical simulation of a chemotaxis model of the form given by equations (39) and (40). Specifically, we take [81, 82]

$$
\begin{aligned}
& \frac{\partial n}{\partial t}=\nabla \cdot(D \nabla n)-\nabla \cdot(\chi n \nabla c)+r n(N-n), \\
& \frac{\partial c}{\partial t}=\nabla \cdot(\nabla c)+\frac{n}{1+n}-c,
\end{aligned}
$$



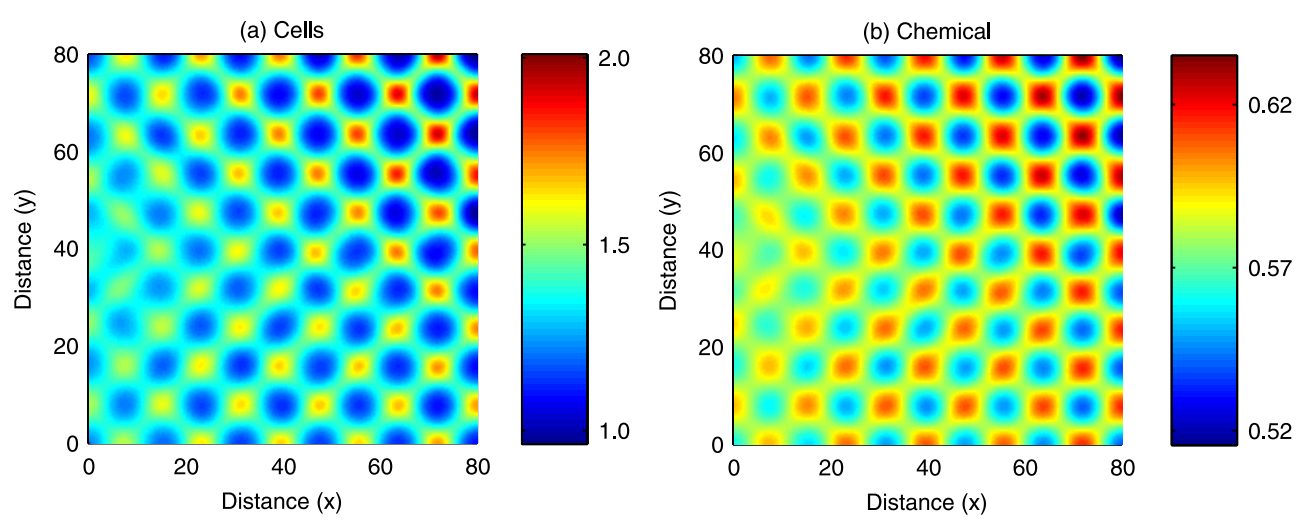

Figure 3. Numerical simulation of a chemotaxis model, given by equations (43) and (44). The field is initially at the homogeneous steady state, with small random perturbations added to the cell density. Diffusion-driven amplification of the heterogeneities results in a regular array of peaks and toughs. Zero flux boundary conditions are imposed, $D=0.25, \chi=1.9, r=0.01$ and $N=1.0$, as in [60]. The models were solved using a finite difference scheme, including complex step differentiation for the Jacobian evaluation [61].
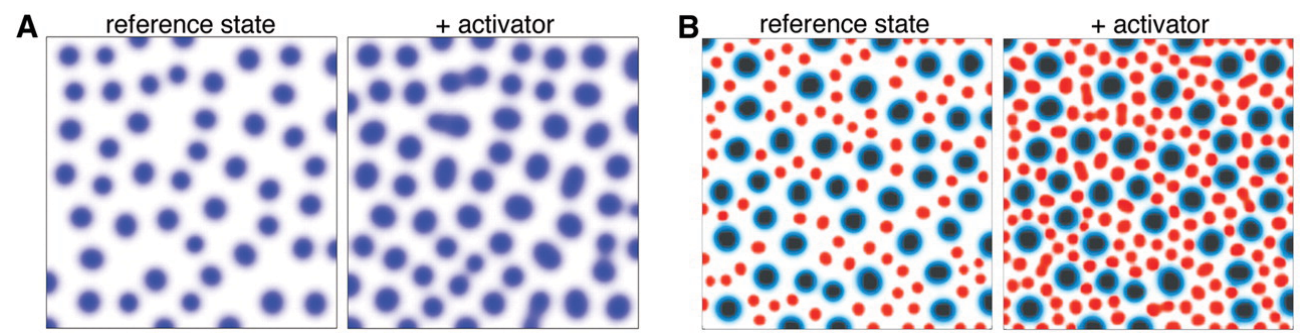

Figure 4. Numerical simulation of a two-stage Turing model for hair follicle formation postulated by Sick and co-workers [8]. (a) primary follicle formation; $(b)$ secondary follicle formation. The blue dots indicate the positions of potential primary follicles whilst the red dots indicate secondary follicles. In each case the left-hand figure shows the WT and the right-hand figure a simulation with increased activator concentration. The dots show areas of high activator concentration. From Sick et al 2006, WNT and DKK determine hair follicle spacing through a reaction-diffusion mechanism Science 314 1447-50. Reprinted with permission from AAAS.

where $D, \chi, r$ and $N$ are all positive parameters. Amplification of small perturbations in cell density across the field leads to a regular array of peaks and troughs in chemical concentration that is mirrored by the cell density pattern.

2.2.2. Mechano-chemical models: examples of taxis models in development. A paradigm of self-organization in the developmental setting is mesenchymal morphogenesis, where the most important cue is considered to be haptotaxis [14,15,32]. Examples have included the emergence of feather buds and limb development. Such models require one to consider how the density of adhesion sites evolves with cell migration, which is typically assumed to scale with the density of the extra-cellular matrix (ECM, a dense mesh of interconnected macromolecular fibres). Hence such models go one step further than those already described; they also have to track the stresses and strains of this hybrid material and the cells that bind to it. 
Consequently, a typical framework for such models is [14-16]

$$
n_{t}=-\nabla \cdot \boldsymbol{J}+r n(N-n),
$$

where $r n(N-n)$, with $r$ and $N$ positive constants, represents logistic growth for cell species $n$ and the flux $\boldsymbol{J}$ is given by

$$
\boldsymbol{J}=-D \nabla n+n \boldsymbol{v}_{t}+a n \nabla \rho .
$$

In the above $v$ is the material displacement of the tissue from its equilibrium, $\rho$ is the density of ECM, $D$ is a cell diffusion coefficient and $a$ is a haptotactic coefficient. Of course, any other taxis form can be included in the flux, if merited by the biology. In addition, one has to close the system by considering the solid mechanics governing the evolution of the displacement field $v$ and the ECM density. Typically the ECM is considered to be convected with the tissue displacement, without decay or growth, yielding

$$
\rho_{t}+\nabla \cdot\left(\rho \boldsymbol{v}_{t}\right)=0 \text {. }
$$

In contrast, the displacement field's evolution is more complicated, governed by a balance of forces given that inertia is unimportant at this scale. Hence one has

$$
\nabla \cdot\left(\sigma_{\mathrm{ECM}}+\sigma_{\text {cell }}\right)+\boldsymbol{F}=0 .
$$

Here, $\sigma_{\mathrm{ECM}}, \sigma_{\text {cell }}$ are, respectively, the ECM and cellular contributions to the stress tensor and $\boldsymbol{F}$ is an external force, if present. In the simplest approximations the ECM is treated via a linear viscoelastic constitutive relation, thus relating $\sigma_{\mathrm{ECM}}$ to $\boldsymbol{v}$ and its temporal derivatives. The cell contribution to the stress tensor, $\sigma_{\text {cell }}$, is modelled phenomenologically in terms of the traction forces exerted by the cells via

$$
\sigma_{\text {cell }}=\frac{n}{1+\lambda n^{2}}\left[\tau \rho+\lambda \nabla^{2} \rho\right]
$$

The stress due to cells, at low cell density, is proportional to the density of cells, $n$, and the ECM density, $\rho$, and decays at larger cell densities due to contact inhibition; $\tau, \lambda$ are simply constant coefficients. Finally a non-local term, $\nabla^{2} \rho$, is a leading order correction due to the fact that the forces exerted by a cell are proportional to the number of focal adhesions in contact with the cell, namely, $\int_{\text {cell }} \rho \mathrm{d} \boldsymbol{x}$. The Laplacian emerges as the leading order correction on expanding the integrand about the centroid of the (assumed isotropic) integration domain; the fact that the cell size is far smaller than other model length scales implies that higher order corrections are certainly negligible.

Equations (45)-(49) are a closed system once supplemented with an expression for external forces, $\boldsymbol{F}$, the domain plus initial and boundary conditions. Such a system constitutes a mechano-chemical model and simultaneously tracks cell movements and their response to haptotatic gradients.

2.2.3. Fluid advection models. In fluid advection models, the cells are surrounded by a fluid whose dynamics cannot be decoupled from the biology, akin to the coupling of solid mechanics and cell motility in the mechano-chemical models. An important example in development is the breaking of left-right symmetry which is species-dependent. In mouse, the most commonly studied mammalian embryo, fluid flows induced by the motion of primary cilia within a sealed depression formed at the ventral side of the primitive streak, the embryological node, are implicated in left-right symmetry breaking. The conversion of this nodal flow into a symmetry breaking signal has yet to be fully explained, though numerous hypotheses are being considered. Simulations of these unsteady flow fields and transport within them assist in the understanding and testing of these hypotheses, an area of intense active research reviewed elsewhere $[2,3]$. 


\subsection{Illustrating comparisons between experiment and modelling}

The application of morphogen models to Drosophila patterning is widespread; an approach employed by Eldar and co-workers [83] used PDE models to investigate the robustness of gradients in the wing disc. Combined studies involving model-building and experimentation were used to explore feedback loops for ligand degradation. Numerical screens for robust networks were able to identify self-enhanced ligand degradation as a possible underlying mechanism, and experimental observations are consistent with this analysis.

One of the most successful examples of the application of Turing's model to a biological system comes from the work of Sick and co-workers [8] on hair follicle spacing. The authors postulate that a two-stage reaction-diffusion mechanism underlies the periodic appearance of hair follicles in mouse. A first wave of patterning establishes an array of primary follicles that then directs formation of the secondary follicles by controlling production terms in the model. Predictions from the model, such as over-expression of the activator leading to increased follicular density, were verified experimentally, thus providing increased support for a Turing mechanism underlying this process. Figure 4 shows the results of numerical simulation of both primary and secondary stages of the modelling process, with predictions from activator over-expression also shown.

Asai and co-workers hypothesized that a Turing model underlies the formation of pigment patterns on the skin of the angelfish Pomacanthus and zebrafish [26,84]. By varying a single parameter in a simple reaction-diffusion model, they were able to obtain a variety of patterns found to occur in wild type and mutant lines; illustrative results of this study are shown in figure 5 for zebrafish. The authors are also able to suggest that the leopard gene may regulate the kinetics of activator synthesis-cloning and molecular analyses are now needed to provide a full interpretation of their results.

Miura and co-workers [62] applied Turing's model to a slightly more complex case: they considered digit formation on a growing domain and showed that a simple reaction-diffusion system can reproduce the patterns observed in Doublefoot mice by assuming saturation of the activator kinetics.

There are, however, cases in which such models have been proven incorrect for modelling self-organization. The striped expression of Drosophila gap and pair-rule genes seem to be one such example in which the elegant Turing models are inapplicable [85]: specific instructions from each gap gene protein seem to direct the formation of the pair-rule stripes. Nonetheless, such models provide an excellent starting point from which to begin investigating self-organization-hypotheses and ideas which stem from theoretical investigation have the potential to direct experimental research in an evidence-based fashion, an efficient approach given the plethora of possible mechanisms underlying biological phenomena.

\subsection{Model comparisons}

A natural question to ask is whether one can distinguish between these models either mathematically or biologically? Most of the mathematical studies begin with linear analysis which looks for eigenfunctions of the Laplacian and in such cases it is the shape of the domain and boundary conditions that determine the patterning, rather than the details of the model itself. Thus, any differences need to be determined at the nonlinear level and, while there have been quite a lot of studies of Turing and chemotaxis models in the nonlinear regime, little has been done on the mechano-chemical models owing to their complexity [86, 87]. It has been shown that Turing and chemotaxis models can both exhibit spiked solutions and that these can move in space and coalesce [88]. It has also been shown that all these models can 


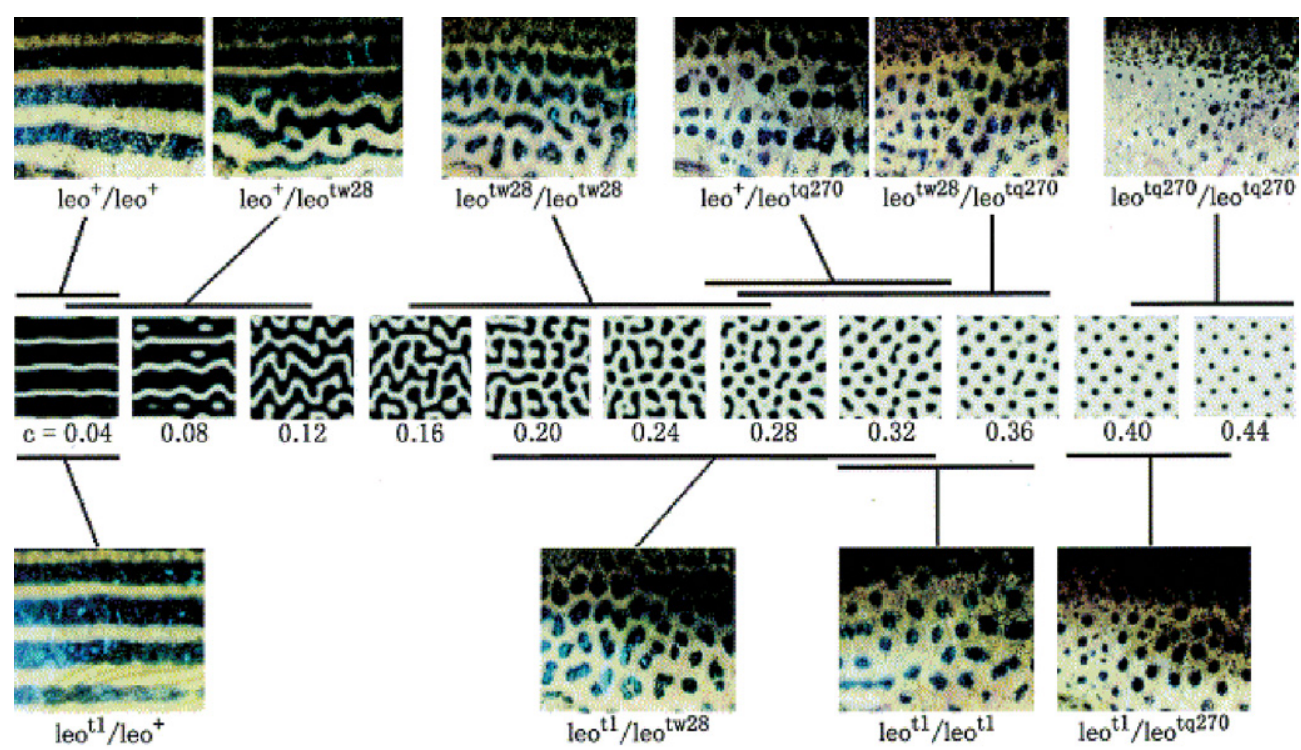

Figure 5. Comparison of numerical simulation of a Turing model for zebrafish pigmentation with biological observations [84]. A range of fish mutant in the leopard gene are shown, with the variation of the pattern from the WT increasing from left to right. The range of patterns can be capitulated by gradual variation in a single parameter of the Turing model. Reprinted from Asai et al 1999, Zebrafish Leopard gene as a component of the putative reaction-diffusion system Mech. Dev. 89 87-92, with permission from Elsevier.

exhibit travelling waves if a disturbance is initiated at one end of the domain and patterns, including chaotic-like instabilities, can be left in the wake of the propagating front [89-91]. This phenomenon is discussed in more detail in section 2.4.1. Given that patterns emerge from these models due to the same principle of short-range activation, long-range inhibition, it is hardly surprising that it is difficult to distinguish between them.

From a biological point of view it is difficult in many cases to determine if pattern arises due to a chemical pre-pattern or due to cell condensation (aggregation) because at the moment it is very difficult experimentally to distinguish between cause and effect. However, there are examples of where this has been done [92].

2.4.1. Propagating patterns. Many patterning processes in biology take place behind travelling fronts. For example, feather bud formation in vivo takes places sequentially: on the dorsal tract (as shown in figure 1) an initial row of feather buds forms along the head-tail axis and subsequent rows form in an ordered fashion, propagating out from the midline [93, 94]. As mentioned in section 2.4, each of the models described here is able to display propagating pattern formation, via initial conditions involving a localized perturbation to the homogeneous steady state. In order to illustrate this result, figures $6(a)$ and $(b)$ show the results of simulating the cell-chemotaxis system given by equations (43) and (44) with the fields initially at the homogeneous steady state and a perturbation added to the cell density at the origin.

However, these types of models, with initial conditions perturbed locally about a steady state, are unsuitable for modelling the majority of biological systems due to their inherent inability to buffer against perturbations. Any perturbation in constituent densities 

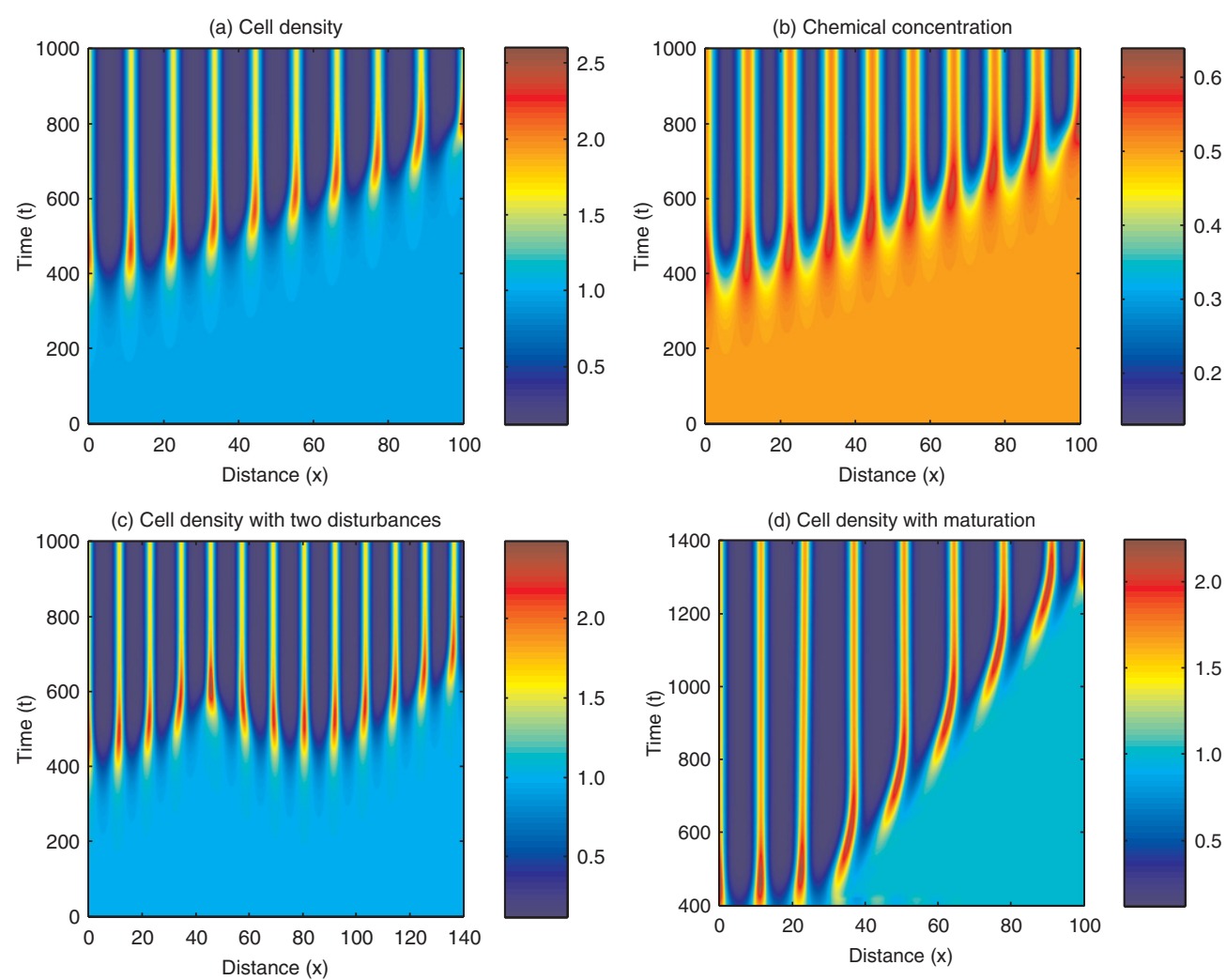

Figure 6. Propagating patterns arising from the chemotaxis system given by equations (43) and (44). (a), (b) Cell density and chemical concentration over time. The field is initially at the homogeneous steady state, with small random perturbations added to the cell density around $x=0$. We see a pattern of regions of high cell density/chemical concentration interspersed with regions of low cell density/chemical concentration propagating out from the point of initial disturbance. (c) Disturbances to the steady state cell density at both $x=0$ and $x=80$ initiate two propagating waves of patterning. (d) Addition of a maturation parameter $\chi \mapsto \chi H(c t-x)$ slows the natural rate of patterning and also makes the field ahead of the pattern stable to perturbation. In each case zero flux boundary conditions are imposed, $D=0.25, \chi=1.9, r=0.01, c=0.08$ and $N=1.0$, as in [60], and the models were solved using the MATLAB function pdepe.

or concentrations away from the initial point of disturbance would cause secondary waves of patterning to propagate from the sites of perturbation, interacting with the initial pattern and destroying the directionality of pattern formation. Figure 6(c) shows the results of adding a second initial perturbation to the cell-chemotaxis system; two waves of patterning arise in this case.

Within the context of these types of PDE models for pattern formation, one can achieve stability to such types of perturbation, whilst ensuring that sequential patterning takes place, by assuming that a bifurcation parameter changes spatio-temporally. For example, figure $6(d)$ shows the results of numerical simulation of the same cell-chemotaxis system with the chemotaxis parameter $\chi \mapsto \chi H(c t-x)$. In this way, cells in more distant regions of the field gain the ability to respond to chemotactic signals later than those situated closer to $x=0$. In the case shown, the natural speed of pattern propagation is restricted by the rate at which cells become able to react to gradients in chemical concentrations and the wavelength of the pattern is also increased. 

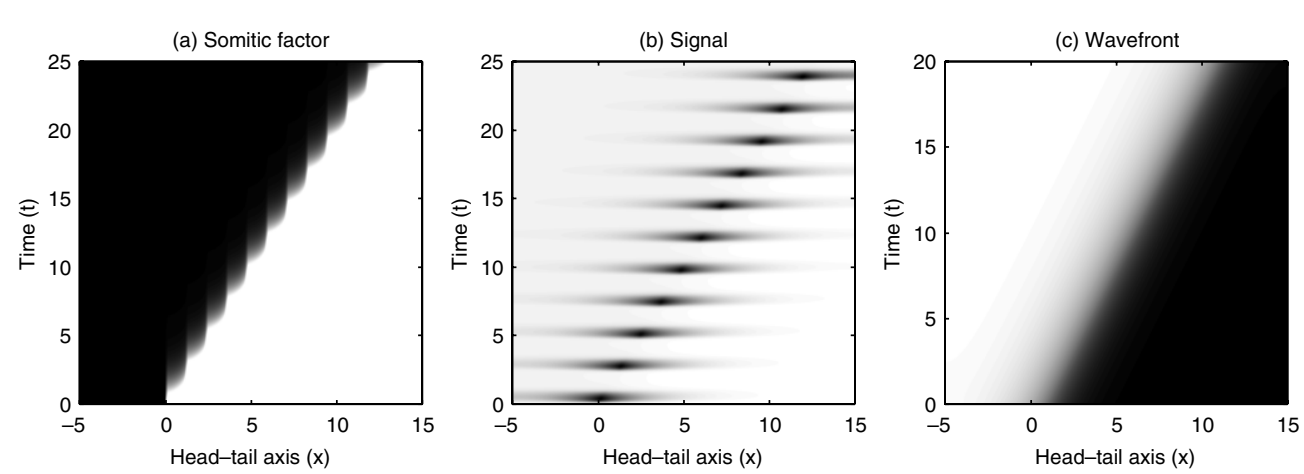

Figure 7. Propagating patterns of somite formation produced by the model of Baker and coworkers $[13,95,96]$. Regression of a wavefront along the head-tail axis causes a series of discrete signals that cause corresponding switches in the levels of somite factor from low to high. In each case dark areas indicate areas of high concentration.

Another method for achieving such kinds of propagating patterning is to assume that the system is bi-stable and that a propagating parameter switches the system from one state to another in a discrete manner. This idea has been used by Baker and co-workers [13,95, 96] in the context of somitogenesis, segmentation of the head-tail axis of vertebrate embryos (see figure 1). In these models, the expression of a high level of a 'somitic factor' causes cells to form somites. Bi-stable kinetics coupled with a wavefront which enables cells to switch from a low to a high level of somitic factor, via interaction with a signalling molecule, results in a propagating pattern of somitic factor concentration. Figure 7 shows the results of numerical simulation of the model of Baker and co-workers $[13,95,96]$. These models do not suffer from the problem mentioned above because, by the nature of the bi-stable kinetics, small perturbations are damped.

\subsection{Outlook}

The above models are, mainly, at the macroscopic, coarse-grained level and their principal aim is to show how patterns can emerge from the combination of non-patterning individual behaviour. They recognize that biological functions/structures arise from an integration of more basic functions, something that is only now being seen to be true by the wider community. For their time, they were adequate in that the prevailing biological data were also at the macroscopic coarse-grained level. In fact, they can still be considered to be very useful paradigm models for patterning on the macroscopic scale, either alone or coupled together [22, 60, 97, 98].

The revolution in molecular biology has led, however, to very different types of data and therefore if models are to make an impact in the biological sciences they must be formulated at the level of the data. These data are, increasingly, at the fine-grained molecular level and therefore models have to take into account the effect of small numbers (where deterministic approaches may be invalid and stochastic approaches more appropriate), obstacles within and tortuosity of tissue (calling into question the assumption of simple Fickian diffusion) and incorporate cell-cell signalling and cell-chemical responses into higher (tissue) level approaches. We now consider some of the open questions that arise as a result of trying to incorporate these phenomena into models. 


\section{Open problems and future challenges}

Experimentation is yielding enormous amounts of information concerning the network of biological interactions underpinning the emergence of structure, plus detailed spatial and temporal data mapping gene expression and cell location. In the following we consider the revolution emerging from molecular biology and biological imaging, plus the challenges and opportunities this presents to the modelling community.

\subsection{Molecular biology and self-organization}

Experimental advances entail that the molecular mechanisms underlying self-organization in developmental biology are being elucidated. Such developments impact throughout the area, providing new data, rejecting old hypotheses and highlighting where further work is required. Theoretical models need to keep pace to continue to achieve their objective of guiding insight, suggesting empirical tests of hypotheses and providing a unique, and complementary, means of probing our understanding of this complex biological field.

As an example of how novel data are rejecting old hypotheses, it was often assumed that the 'activation' required for a Turing system may arise from a protein inducing the release of a bound version of itself from cell membranes [58]. However, a common experimental technique, in situ hybridization, has been developed to track and visualize the local concentration of specific mRNA transcripts throughout a tissue, thus indicating transcription. In systems where there is a putative Turing mechanism, such as with zebrafish mesoderm cell fates [6] or avian bud formation [21], the nominal activator is tracked in this manner. This indicates that the self-induced production of the activator is actually via gene expression, which has a substantial impact on modelling formulation, as we now discuss.

3.1.1. Gene expression time delays. Experimental observations show us that gene expression takes time; any delays associated with signal transduction will simply extend this delay. Exactly how much time depends on the size of the genetic sequence to be expressed. Estimates range from just over ten minutes to several hours $[99,100]$. On the other hand, patterning times in developmental processes have been estimated to be as low as 20 min [55]; a gene expression time delay is thus likely to be highly significant for the fastest developmental events. Such observations are exacerbated by the fact that incorporation of a gene expression time delay in a simple Turing system reveals that time delay can induce a disproportionate retardation, much greater than the gene expression delay, in the time to patterning and, on growing domains, can cause patterning failure [101]. Thus the effects of the time delay are far from trivial. These results are illustrated in figure 8 where a selection of numerical simulations from the paper of Gaffney and Monk [101] are presented. For exponential domain growth, with a doubling time of two days, the authors were able to show that even relatively small delays greatly influenced the patterns formed, with eventual failure for longer, though not unreasonable, delays.

Any self-organizing system reliant on gene expression for structure formation on time scales which are not substantially greater than the gene expression time delay is potentially susceptible to the effects of this delay. Due to the need for activation this includes putative Turing systems. Modelling opportunities arise in investigating current models to ascertain whether gene expression delays have substantial effects. Numerous further questions arise. For example, can mathematical modelling classify when gene expression time delays may be neglected in a given pattern forming system? Will a gene expression time delay induce oscillations should any gene products form a negative feedback loop? Are the stability and 

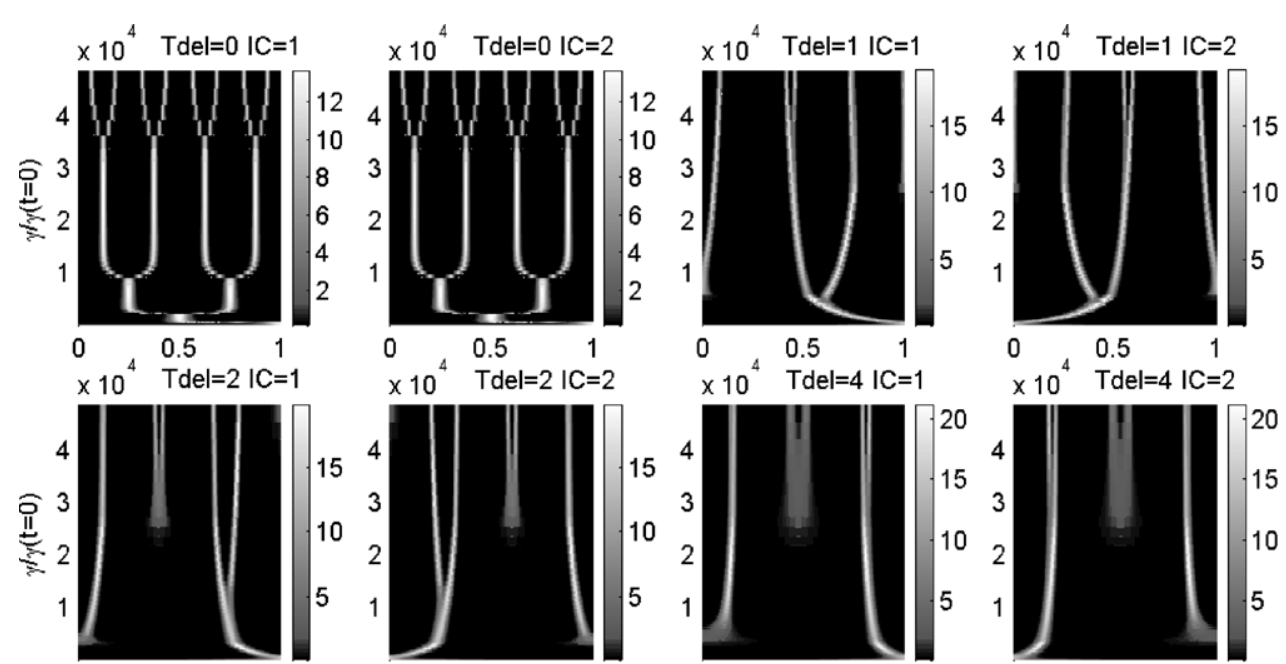

$\times 10^{4} \quad$ Tdel $=2 \mathrm{IC}=2$
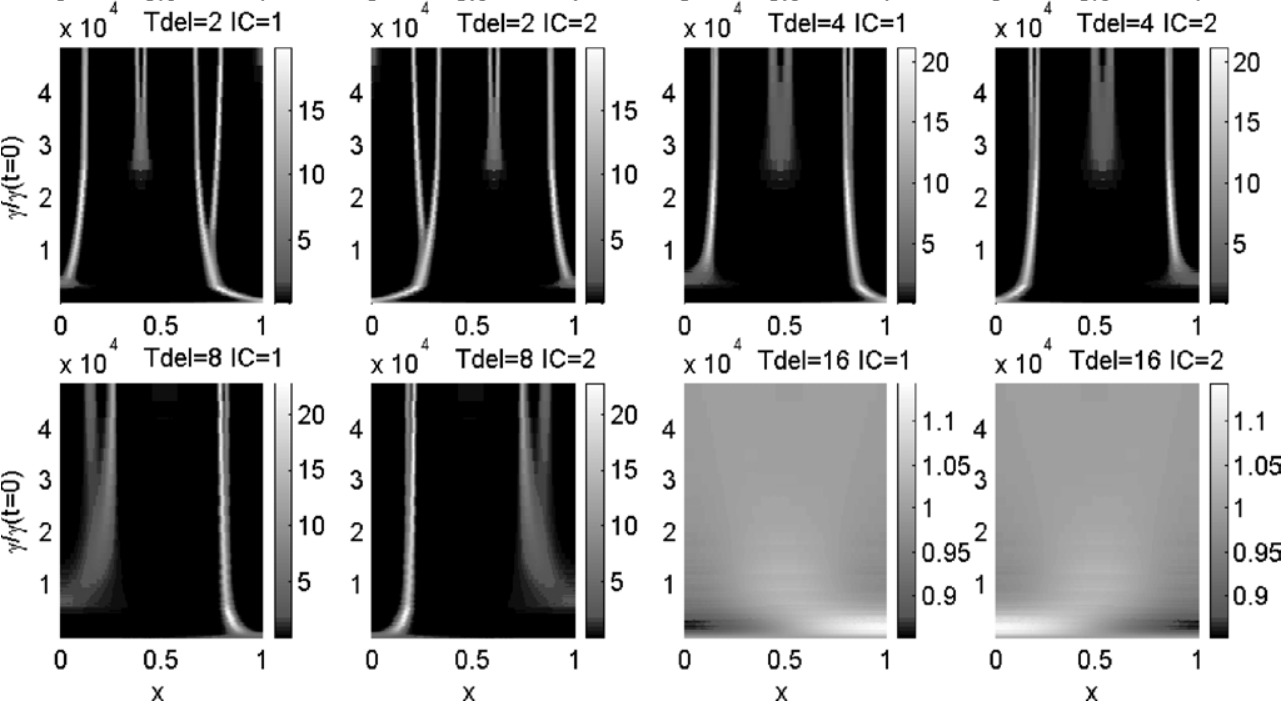

Figure 8. Results of numerical simulations of a Turing reaction-diffusion system with domain growth and delays [101]. In each case, the concentration of activator is plotted. The domain doubling time is 2 days and the time delays are marked on each individual plot in units of $12 \mathrm{~min}$. The vertical axis is the (square) domain length, which is monotonically increasing in time, and the horizontal axis is the spatial coordinate of the simulations. Tdel denotes time delay and IC the initial conditions. It is easy to see that, for both sets of initial conditions (given in [101]), time delays have a marked effect on the pattern formed, with eventual failure. Reprinted from Gaffney and Monk 2006 Gene expression time delays and Turing pattern formation systems Bull. Math. Biol. 68 99-130, with permission from Springer.

robustness of pattern forming systems altered by such gene expression delays? What happens when time delays are considered within a large network of interacting morphogens?

3.1.2. Networks, systems and synthetic biology, parameter estimation. Typically, the modelling of pattern formation in biology has relied upon putative models with a small number of interactions, with the canonical example of two morphogens in Turing's model. This was reasonable before the revolution in molecular biology; however, to be realistic in this day and age, models must be more detailed as substantially more information is available for utilization and investigation in biological modelling. An informative example is the study of a model of gene product interactions, as illustrated in figure 9, in the determination of segment polarity in Drosophila [102]. With over 100 ordinary differential equations (ODEs) and 48 adjustable parameters, it was still possible to use modelling tools to demonstrate that the current network representing gene interactions could not explain observed patterning events but simple additions to the network could. The inability to estimate parameters was compensated for by 


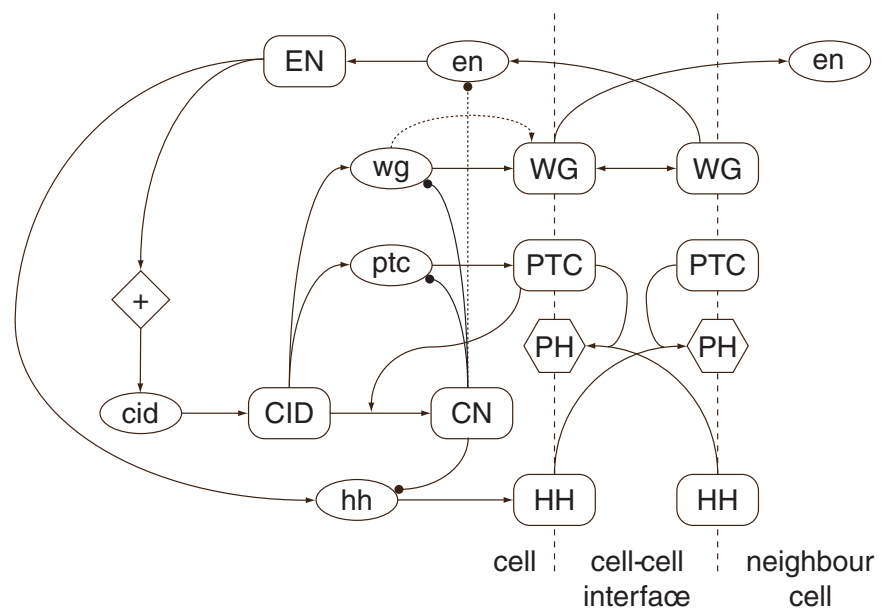

Figure 9. A schematic of the gene product interaction network considered in [102]. The solid lines indicate known interactions whilst the dashed lines indicate interactions suggested by numerical simulation of the network. WG, wingless; EN, engrailed; HH, hedgehog; CID, cubitus interruptus (whole protein); $\mathrm{CN}$, repressor fragment of cubitus interruptus; PTC, patched; $\mathrm{PH}$, patchedhedgehog complex. Ellipses, mRNAs; rectangles, proteins; arrows, positive interactions; circles, negative interactions. ci is basally expressed (+ in rhombus). Reproduced from [102] with slight modifications.

numerical simulations using $\mathcal{O}(250000)$ random selections from the extremely large regions of feasible parameter space-the vast majority of these random selections yielded pattern formation similar to that observed in situ.

This highlights that modellers should be alert to the fact that patterning events may depend on the subtle details of network interactions and that self-organization models based on a small number of interacting chemicals should often, but perhaps not always, be superceded with ones including the effects of additional interactions as data become available. However, this is where a balance must be struck between biological accuracy and the ability of the modeller to fully explore the system using both analytical and numerical tools. Such comments are not reserved for development either. The networks underpinning signal transduction in bacterial chemotaxis are also being elucidated [103], and modelling frameworks are being developed to incorporate such information into macroscale PDE models [80].

While molecular biological methods often reveal interactions, quantitative estimates of reaction rates, for example, are rare. This is problematic, especially for moving in the direction of larger models. Qualitative techniques will be especially valuable for such studies. For example, graph theory can be applied to study the stability of large networks of ODEs [104, 105]. Note that the applied study of the linear stability of PDEs typically involves the reduction to ODEs via an expansion in the eigenfunctions of the transport operator. Thus graph theoretic techniques are applicable in the context of biological networks with pattern forming properties in revealing aspects of stability properties from network topologies rather than network dynamics.

One can also adopt the approach of performing a random sampling of parameter space [102]. This can be highly revealing even when an exhaustive sampling is impossible, and further opens up the possibility of examining the role of biological networks in patterning. The latter task, which will be enormously demanding computationally, emphasizes the vital exercise 


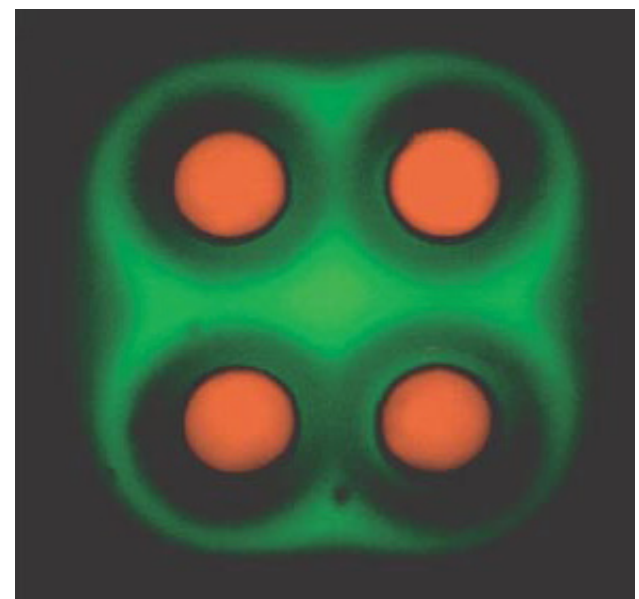

Figure 10. An example of a cell differentiation pattern with one cell type expressing green, the other red, fluorescent protein, emerging from an artificial self-organizing system. Reprinted by permission from Macmillan Publishers Ltd, Basu et al 2005, A synthetic multicellular system for programmed pattern formation Nature 434 1130-4, copyright 2005.

of devising accurate asymptotics for larger scale networks, even if the goal is to speed up numerical calculations rather than to write down analytical solutions. This is certainly possible with biological systems as large as those envisaged even in the current context [106-108].

In addition, while parameter estimates can be rare, they should be considered when available. Fluorescent recovery after photobleaching (FRAP) techniques, for example, have become widely available for measuring diffusion coefficients in realistic environments [109, 110]. Sometimes parameter estimates can highlight modelling difficulties, as with our previous observations that in the absence of large diffusivity ratios for putative Turing morphogens, there is unreasonable parameter fine-tuning. However such difficulties often present novel opportunities. In the current context, the complexity of both networks and the cellular microenvironment can allow Turing-style patterns without parameter fine-tuning. For example, a model with more than two reactants has successfully shown a Turing instability with equal diffusion coefficients for ATP and ADP given realistic glycolytic reaction rates [111]. Analogous comments apply for pattern formation models considering the intraand extracellular environments coupled via signal transduction [112]. These exemplify how the additional biological phenomena discussed in this section, and below, represent potential resolutions of fundamental and paradoxical difficulties in proposed mechanisms of biological self-organization, rather than distracting and unnecessary complications.

As a final motivation for larger scale models, we mention an exciting experimental development, namely, the prospect of synthetic multicellular systems for artificial pattern formation [113]. While prototypical, such studies illustrate how genetically engineered receiver cells can be designed to sense a chemical gradient and undergo a desired pattern formation-an example of this is shown in figure 10. Highly amenable to mathematical modelling, pattern formation by design has broad potential application areas including tissue engineering, biosensing and bio-directed fabrication [113]. Such systems will be substantially more complicated than a Turing pair however. For example, a prospective mechanism for sharpening the edges of artificial patterns could make use of the biological mechanisms of segment polarity in Drosophila [113], requiring $\mathcal{O}(100)$ equations for the relevant network interactions [102]. 
Thus, in summary, a major challenge in the modelling of biological pattern formation is to consider coupling of large networks, with significantly more than two interacting proteins, plus subcellular dynamics, to support understanding not only of biological systems but also in the context of synthetic biological design.

\subsection{Noise and robustness}

A fascinating advance in molecular biology is the development of in vivo genetic reporter systems, that is the genetic expression, and subsequently tracking, of fluorescent proteins at the cellular level, as illustrated in our discussion on synthetic biology. This technique additionally has allowed the investigation of noise in the gene expression underlying biological self-organization processes such as embryonic development [114-116]. Noise is observed to be present in general with two types of source: (i) intrinsic variability caused by, for example, fluctuations in promoter activation and deactivation, mRNA and protein production and decay; (ii) extrinsic variability, defined as 'fluctuations and population variability in the rate constants associated with these events' [117] and typified by changes in temperature and cellular conditions. Robustness is usually defined as the ability of a system to buffer against such fluctuations, so that a mechanism may reproducibly deliver a required output. It is exemplified by the fact that, for most genes, heterozygous pairs do not lead to an apparent phenotype [64].

One may subsequently speculate that noise on the individual cell level will not impact on modelling at larger scales, given that even small biological systems consist of substantial cell numbers. However, numerous individual-based stochastic effects manifest themselves at higher scales, and an open area of research is the understanding of when such cellbased fluctuations are indeed negligible. Here we outline two examples where they are not: (i) stochastic-induced switching between stable steady states in a bi-stable system; (ii) errors in differentiation signals inherited from the effects of fluctuations on morphogen gradients.

Stochastic switching. We demonstrate the effects of stochasticity upon bi-stable systems using an example presented by Erban and co-workers [118]: consider the following scheme of reactions for a chemical $A$,

$$
2 A \stackrel{k_{1}}{\rightarrow} 3 A, \quad 3 A \stackrel{k_{2}}{\rightarrow} 2 A, \quad \emptyset \stackrel{k_{3}}{\rightarrow} A, \quad A \stackrel{k_{4}}{\rightarrow} \emptyset .
$$

One may write down a simple ODE to describe the system using the law of mass action [119]:

$$
\frac{\mathrm{d}[A]}{\mathrm{d} t}=-k_{2}[A]^{3}+k_{1}[A]^{2}-k_{4}[A]+k_{3},
$$

where $[A]$ denotes the concentration of $A$. It is easy to see that the system, given the parameter values detailed in the caption of figure 11, has two stable steady states, given by $[A]_{s}=100,400$, and one unstable steady state, given by $[A]_{u}=220$. Analysis of the system shows that phase trajectories with $[A](0)<220$ will converge to the lower steady state, whilst those with $[A](0)>220$ will converge to the upper one. The system may, however, be reinterpreted as a continuous time Markov chain and investigated using the Gillespie stochastic simulation algorithm (SSA) [120]. A very different behaviour is observed-stochastic effects allow the system to 'jump' between the two steady states.

The results of two realizations of the system are shown in figure 11. The long time scale simulation allows us to see switching between the two stable steady states, as a result of the inherent stochasticity in the system - this is not, by contrast, achievable with the deterministic PDE model. 

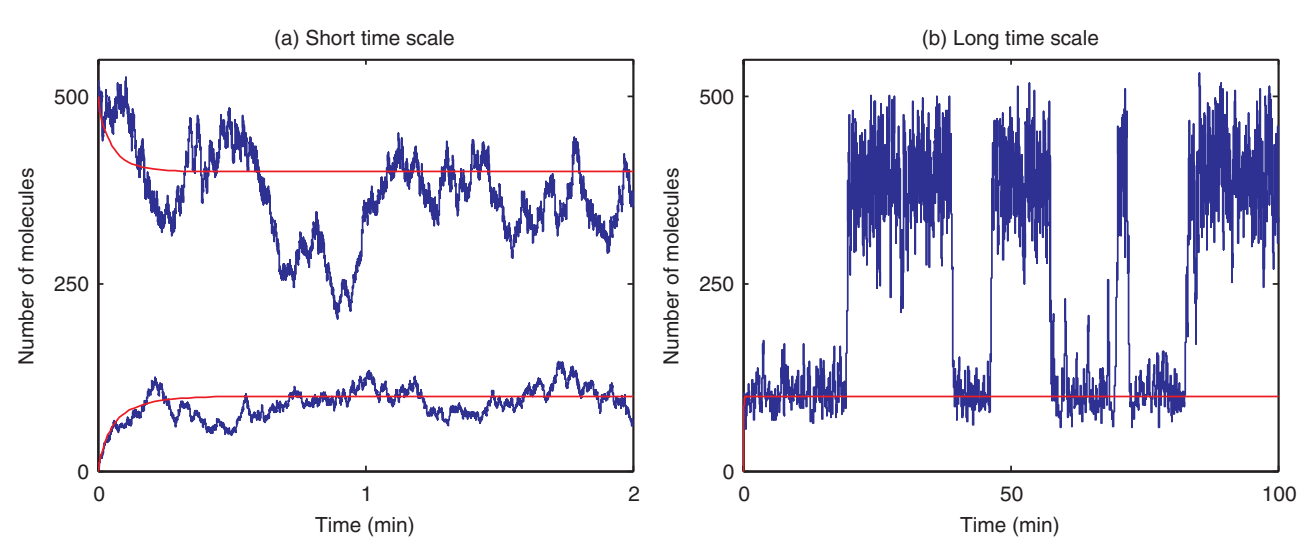

Figure 11. Numerical simulation of the chemical scheme (50) using both a deterministic and stochastic approach. In both figures the results of numerical solution of equation (51) are shown by the red, smooth line whilst results of a stochastic realization are shown by the blue, jagged line. (a) Over short time scales the system tends to remain close to the steady state near which it started; $(b)$ over longer time scales we observe switching between the steady states in the stochastic simulation. Numerical simulations were based on the code associated with [118], found at http://www.maths.ox.ac.uk/cmb/education/. Parameters are as follows: $k_{1}=0.18 \mathrm{~min}^{-1}$, $k_{2}=2.5 \times 10^{-4} \mathrm{~min}^{-1}, k_{3}=2200 \mathrm{~min}^{-1}, k_{4}=37.5 \mathrm{~min}^{-1}$.

Morphogen gradients: the stochastic French Flag model. Diffusion can also be represented in a stochastic manner. Consider, for example, the system

$$
\frac{\partial u}{\partial t}=D \nabla^{2} u-\lambda u
$$

in one spatial dimension, where $u$ represents the morphogen concentration, $\lambda$ the rate of linear decay and $D$ the diffusion rate of morphogen. Numerical simulation of the system for $x \in[0,1]$ with a flux of morphogen at the left-hand boundary and reflecting conditions at the right-hand boundary is shown in figure $12(a)$. A steady gradient is formed which decreases monotonically over the domain. The idea behind Wolpert's French Flag model [45] is illustrated in the figure: cells on the domain which experience $u \geqslant 60$ become 'blue', those with $30 \leqslant u<60$ become 'white' and those with $u<30$ become 'red'.

Figure $12(b)$ shows the result of simulating the same system using a Gillespie approach [120]. The idea involves dividing the domain up into $k$ equally sized boxes such that the following diffusion 'reactions' take place:

$$
u_{1} \stackrel{d}{\rightarrow} u_{2} \stackrel{d}{\rightarrow} \cdots \stackrel{d}{\rightarrow} u_{k-1} \stackrel{d}{\rightarrow} u_{k}, \quad u_{k} \stackrel{d}{\rightarrow} u_{k-1} \stackrel{d}{\rightarrow} \cdots \stackrel{d}{\rightarrow} u_{2} \stackrel{d}{\rightarrow} u_{1},
$$

together with the flux and decay equations

$$
\emptyset \stackrel{r}{\rightarrow} u_{1} \quad \text { and } \quad u_{i} \stackrel{\lambda}{\rightarrow} \emptyset, \quad \text { for } i=1, \ldots, k,
$$

where $u_{i}, i=1, \ldots, k$, represents the number of morphogen molecules in box $i$. The diffusion constants $D$ and $d$ are related via the expression $d=D /(\Delta x)^{2}$, where $\Delta x$ is the box width. We see that there are several boxes which become 'incorrectly' specified via the stochastic realization, compared with the deterministic PDE description.

Given the phenomena just outlined, we now proceed to consider another active research field, addressing how such noise may be buffered. We outline some of the mechanisms proposed to increase robustness and include modelling-based insights. We then move to 

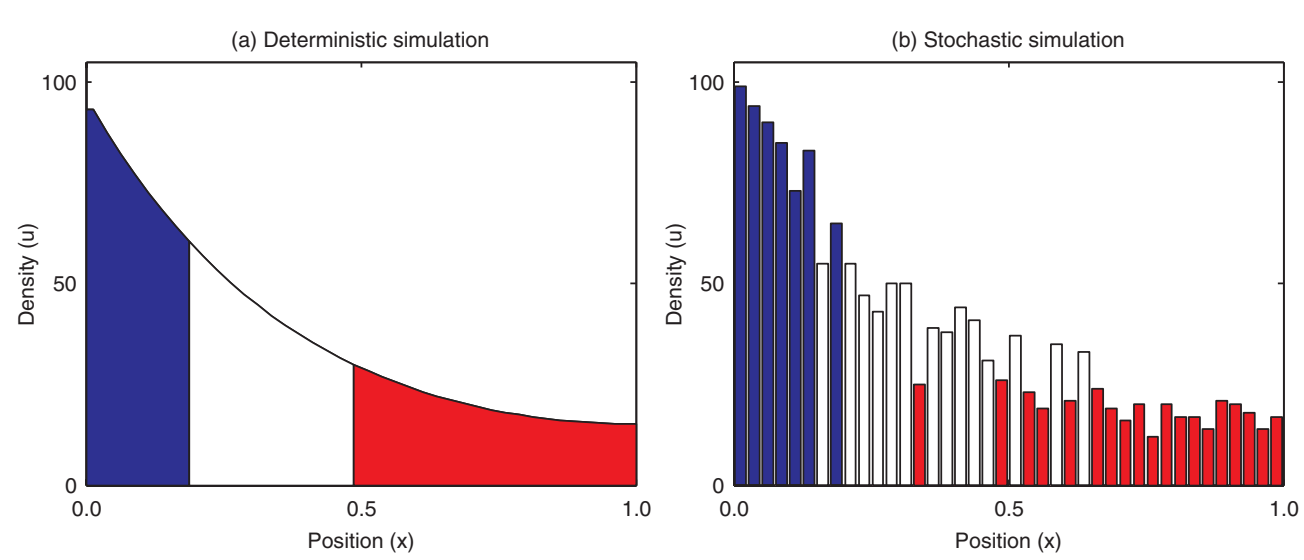

Figure 12. Numerical simulation of diffusion using both a PDE formulation and a Gillespie approach [120]: (a) numerical solution of equation (52) using a finite difference scheme; (b) stochastic realization of equations (53) and (54). In each case, concentrations of $u=60$ and $u=30$ denote thresholds for different differential programs (blue (left-most), red (middle) and red (right-most)). Numerical simulations were based on the code associated with [118], found at http://www.maths.ox.ac.uk/cmb/education/. Parameters are as follows: $D=0.0625, r=6.0$, $\lambda=0.004$ and $\Delta x=0.025$.

modelling frameworks which can integrate stochastic effects with the PDE modelling of biological systems.

3.2.1. Mechanisms for buffering against noise. No matter how well regulated, any biological system is still influenced to some degree by stochastic effects and a number of theories have been put forward to explain the apparent robustness of systems to such noise. For example, the existence of large gene networks has been postulated to improve robustness by incorporating feedback loops that buffer against fluctuations and also by allowing for redundancy. However, the mechanisms which buffer against fluctuations to yield robustness, especially in fields such as developmental biology, are still a subject of much debate. Here we outline a few examples where modelling has been used to demonstrate the ability of systems to filter out noise or to postulate a mechanism to increase robustness to the effects of noise.

Tostevin and colleagues [121] have recently argued, using mathematical techniques, that there are fundamental limits to position determination by morphogen gradients. They propose that time-averaging of a single gradient can lead to great precision even in the limit of very low protein copy numbers and that there exists an optimal length scale for the gradient in order for precision to be achieved. Dessaud and co-workers [46] show that some form of temporal averaging is at work during chick limb specification; they show that changing the time and level of exposure to Shh have equivalent effects on intracellular signalling. However, that temporal averaging is employed to increase robustness to fluctuating signals is yet to be definitively demonstrated.

A textbook example of the ability of a developmental system to buffer against fluctuations comes from the work of Kruse and co-workers [122-124]: they investigated the roles of diffusive versus transcytotic trafficking during morphogen gradient formation in the Drosophila wing disc. As a result the authors suggest, via a combination of mathematical and theoretical techniques, that repeated internalization and externalization of the ligand Dpp provides a more stable mechanism for gradient formation by buffering against variability in the flux of Dpp 

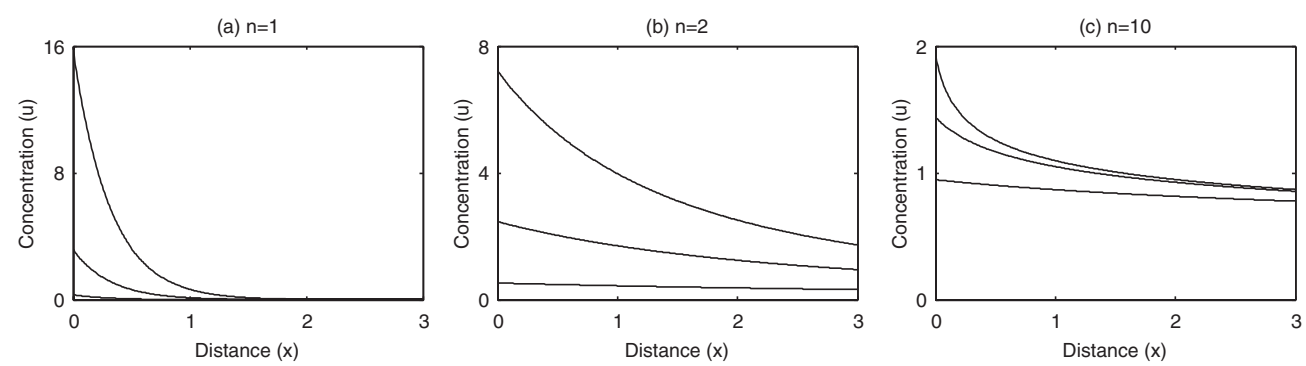

Figure 13. Steady state morphogen profiles for different types of decay and morphogen flux, $f$. (a) Linear decay, $n=1$, equation (56), results in a profile that changes markedly. $(b)$, $(c)$ as the decay rate becomes increasingly nonlinear, equation (57), the profile becomes increasingly robust to changes in $f$. Parameter values are as follows: $D=1.0, \lambda=0.1, f=0.1,0.5,5.0$.

into the wing disc. We also note that the interaction of multiple gradients has been proposed as a model mechanism for complex patterning [125, 126].

Self-enhanced ligand degradation. One simple example of the use of mathematical models to suggest robust mechanisms comes from the work of Eldar and colleagues $[64,83]$ into the mechanisms underlying morphogen gradient formation. The authors used a PDE of the form

$$
\frac{\partial u}{\partial t}=D \nabla^{2} u-\lambda(u)
$$

in one spatial dimension to describe the kinetics of morphogen concentration, $u$. A flux of morphogen at the left-hand boundary, together with suitable conditions at the right-hand boundary and an initial distribution of morphogen were also assumed. The authors carried out extensive computational screening of the system using different diffusion and flux rates and assuming different forms for morphogen decay, $\lambda(u)$.

Assuming a linear decay rate, so that $\lambda(u)=\lambda u$, results in a steady state morphogen profile

$$
u_{\mathrm{st}}=\frac{f}{a} \exp \left(-\frac{x}{a}\right), \quad \text { where } a=\sqrt{\frac{D}{\lambda}},
$$

whereas a nonlinear decay rate of the form $\lambda(u)=\lambda u^{n}$ gives

$$
u_{\mathrm{st}}=\frac{\alpha}{(x+\beta)^{m}}
$$

where

$$
m=\frac{2}{n-1}, \quad \alpha=\left(m(m+1) \frac{D}{\lambda}\right)^{m / 2}, \quad \beta=\left(\frac{m \alpha D}{f}\right)^{\frac{n-1}{n+1}} .
$$

$f$ is the flux of morphogen at the left-hand boundary and $x \in[0, \infty)$ such that $u \rightarrow 0$ as $x \rightarrow \infty$.

Figure 13 demonstrates the increased robustness that comes from a nonlinear decay rate. Eldar and co-workers were able to show that this form of self-enhanced ligand degradation is consistent with the properties of the morphogens Wingless and Hedgehog in the Drosophila wing disc [83]. Lander and co-workers have also made substantial contributions in the area, investigating, for example, the roles of diffusion and cell-cell trafficking in morphogen gradient formation $[127,128]$.

Next, we describe three 'error correction' mechanisms [129] that have been suggested for refining tissue patterns established by graded morphogen expression. 
Elimination of mis-positioned cells. This can occur, for example, via increased apoptosis: cells which express markers of a domain other than the one in which they are situated undergo programmed cell death. The phenomenon occurs in Drosophila embryos with altered Bicoid gradients: increased apoptosis in certain regions of the embryos counteracts the effects of gradient alteration $[129,130]$.

Sorting of mis-positioned cells. This may occur, for example, as cells sort according to differences in their adhesive properties $[25,131]$. Hh signalling in the neural tube appears to control cell adhesion properties, thereby allowing the segregation of Hh-responding cells [129, 132].

Re-specification of mis-positioned cells. This occurs, for example, when individual cells are unable to retain their original identity in a group of cells expressing different markers. One documented case of this occurs during inversion of pieces of somite precursor tissue at certain positions along the head-tail axis of vertebrate embryos: normal segmentation results, despite changes in the levels of FGF signalling [133].

These examples suggest not only that the mechanisms underlying development may be robust but also that the embryo may have developed techniques with which to guard against environmental and intrinsic fluctuations.

3.2.2. Incorporating stochastic effects into models. Given that PDE models cannot always be satisfactorily applied to modelling biological systems, we now outline some stochastic methods which could be considered. Our discussion will be motivated by a concrete example: the description of a simple diffusion process. The PDE generally used to describe diffusion of a chemical species $C$ in one space dimension is

$$
\frac{\partial c}{\partial t}=\frac{\partial}{\partial x}\left(D \frac{\partial c}{\partial x}\right), \quad x \in \mathbb{R}, \quad t>0,
$$

where $c$ is the concentration of chemical $C$ and $D$ describes the diffusive flux. The system is closed by specifying conditions as $x \rightarrow \pm \infty$ and an initial condition $c(x, t)=c_{0}(x)$.

Stochastic differential equations. The Langevin form of the diffusive process described above can be written by supposing that the position of each particle evolves according to

$$
X(t+\mathrm{d} t)=X(t)+\sqrt{2 D} \mathrm{~d} W(\mathrm{~d} t)
$$

where $\mathrm{d} W(\mathrm{~d} t)$ is a normal random variable with mean 0 and variance $\mathrm{d} t$ and $D$ is the macroscopic diffusion constant. To simulate the behaviour of $N$ morphogen molecules, a finite time step $\Delta t$ is chosen. The position of each molecule is updated according to the equation

$$
x_{i}(t+\Delta t)=x_{i}(t)+\sqrt{2 D \Delta t} \xi_{i}, \quad i=1, \ldots, N,
$$

where each $\xi_{i}$ is a normally distributed random variable with zero mean and unit variance.

Fokker-Planck equations. The Fokker-Planck equation is used to describe the evolution of the probability density function (PDF) for a particle over time. Let $p(x, t \mid y, s)$ be the probability that the particle is at $x$ at time $t$ under the condition that it was at $y$ at time $s$. Then

$$
p(x, t+\delta t \mid y, s)=\int_{\mathbb{R}} p(x, t+\delta t \mid z, t) p(z, t \mid y, s) \mathrm{d} z .
$$


Multiplying both sides by a smooth test function $\phi(x)$ and integrating over $x$, gives

$\int_{\mathbb{R}} p(x, t+\delta t \mid y, s) \phi(x) \mathrm{d} x=\int_{\mathbb{R}}\left[\int_{\mathbb{R}} p(x, t+\delta t \mid z, t) \phi(x) \mathrm{d} x\right] p(z, t \mid y, s) \mathrm{d} z$.

Using a Taylor expansion on the right-hand side and neglecting terms $o\left((x-z)^{2}\right)$, which we assume to be small, gives

$$
\begin{gathered}
\int_{\mathbb{R}}\left[\{p(z, t+\delta t \mid y, s)-p(z, t \mid y, s)\}+\frac{\partial}{\partial z}\left(\int_{\mathbb{R}}(x-z) p(x, t+\delta t \mid z, t) \mathrm{d} x p(z, t \mid y, s)\right)\right. \\
\left.\left.-\frac{1}{2} \frac{\partial}{\partial z}\left(\int_{\mathbb{R}}(x-z)^{2} p_{(} x, t+\delta t \mid z, t\right) \mathrm{d} x p(z, t \mid y, s)\right)\right] \phi(z) \mathrm{d} z=0
\end{gathered}
$$

Since the above holds for all test functions $\phi$, we have

$$
\begin{gathered}
\{p(z, t+\delta t \mid y, s)-p(z, t \mid y, s)\}=-\frac{\partial}{\partial z}\left(\int_{\mathbb{R}}(x-z) p(x, t+\delta t \mid z, t) \mathrm{d} x p(z, t \mid y, s)\right) \\
+\frac{1}{2} \frac{\partial^{2}}{\partial z^{2}}\left(\int_{\mathbb{R}}(x-z)^{2} p(x, t+\delta t \mid z, t) \mathrm{d} x p(z, t \mid y, s)\right) .
\end{gathered}
$$

Assuming that the diffusion rate is isotropic gives

$$
\int_{\mathbb{R}}(x-z) p(x, t+\delta t \mid z, t) \mathrm{d} x=0,
$$

and letting

$$
\int_{\mathbb{R}}(x-z)^{2} p(x, t+\delta t \mid z, t) \mathrm{d} x=2 D \delta t+o(\delta t)
$$

gives

$$
\frac{p(z, t+\delta t \mid y, s)-p(z, t \mid y, s)}{\delta t}=\frac{\partial^{2}}{\partial z^{2}}(D p(z, t \mid y, s))+o(\delta t) .
$$

Passing to the limit $\delta t \rightarrow 0$ gives the familiar equation

$$
\frac{\partial p}{\partial t}=\frac{\partial^{2}}{\partial x^{2}}(D p)
$$

However, for systems involving more than a few species, the Fokker-Planck equation cannot in general be solved-even numerically. In practice, most researchers will use Monte Carlo methods, solving the Langevin equation many times and using statistical techniques to estimate the PDF.

Chemical master equations. The final approach we consider is that of the chemical master equation-differing in format from the other approaches considered in that it is discrete in space. We divide the real line into boxes of length $\Delta x=1 / k$ so that the number of cells in box $i$ is denoted by $C_{i}$ for $i \in \mathbb{Z}$ and specify the transition probabilities per unit time of a cell moving left or right from box $i$ by $T_{i}^{ \pm}=d$.

In order to construct a master equation describing the evolution of $C_{i}$ we let $P(\boldsymbol{n}, t)$ be the joint probability that $C_{i}=n_{i}$ at time $t$ for $i \in \mathbb{Z}$ and $\boldsymbol{n}$ a vector representing the $n_{i}$. We define creation and annihilation operators $A_{i}^{c}, A_{i}^{a}: \mathbb{R}^{k} \rightarrow \mathbb{R}^{k}, i \in \mathbb{Z}$ and projections $B_{i}: \mathbb{R}^{k} \rightarrow \mathbb{R}$, $i \in \mathbb{Z}$ by

$$
\begin{aligned}
A_{i}^{c}:\left[n_{1}, \ldots, n_{i}, \ldots, n_{k}\right] & \rightarrow\left[n_{1}, \ldots, n_{i}+1, \ldots, n_{k}\right], \\
A_{i}^{a}:\left[n_{1}, \ldots, n_{i}, \ldots, n_{k}\right] & \rightarrow\left[n_{1}, \ldots, n_{i}-1, \ldots, n_{k}\right], \\
B_{i}:\left[n_{1}, \ldots, n_{i}, \ldots, n_{k}\right] & \rightarrow n_{i} .
\end{aligned}
$$


The continuous time evolution of $P(\boldsymbol{n}, t)$ can be written as

$$
\begin{aligned}
\frac{\partial P(\boldsymbol{n}, t)}{\partial t}=\sum_{i \in \mathbb{Z}} d\left\{\left[B_{i} A_{i+1}^{a} A_{i}^{c} \boldsymbol{n}\right] P\left(A_{i+1}^{a} A_{i}^{c} \boldsymbol{n}, t\right)-\left[B_{i} \boldsymbol{n}\right] P(\boldsymbol{n}, t)\right\} \\
+\sum_{i \in \mathbb{Z}} d\left\{\left[B_{i} A_{i-1}^{a} A_{i}^{c} \boldsymbol{n}\right] P\left(A_{i-1}^{a} A_{i}^{c} \boldsymbol{n}, t\right)-\left[B_{i} \boldsymbol{n}\right] P(\boldsymbol{n}, t)\right\} .
\end{aligned}
$$

The stochastic means, defined as $M_{i}=\sum_{\boldsymbol{n}} n_{i} P(\boldsymbol{n}, t)$, are easily established as satisfying

$$
\frac{\partial M_{i}}{\partial t}=d\left(M_{i-1}-2 M_{i}+M_{i+1}\right), \quad i \in \mathbb{Z}
$$

which can be compared with a second order finite difference spatial discretization of equation (59),

$$
\frac{\partial u_{i}}{\partial t}=\frac{d}{\Delta x^{2}}\left[u_{i-1}-2 u_{i}+u_{i+1}\right], \quad i \in \mathbb{Z},
$$

where $D=d /(\Delta x)^{2}$ and $\Delta x$ is the distance between spatial grid points.

As with the Fokker-Planck equation, the master equation formalism is deterministic in the sense that, once the initial probabilities are specified, the future probabilities are fixed [134]. The discrete formulation may be a more accurate representation, especially if concentrations are low. However, it is rare that the master equation is directly simulated as, even for a few species, many hundreds of equations (one for each possible state of the system) need to be simulated. Most often Monte Carlo approaches, such as the Gillespie SSA outlined earlier in section 3.2, are employed.

3.2.3. Open problems concerning noise and biological self-organization. The inclusion of stochastic effects in the modelling of biological systems is a comparatively new field. Hence, there are numerous issues in the area, most of which remain unexplored-here we outline but a few of the questions to which we must begin to seek answers, in order for further understanding in the area.

What is appropriate choice of modelling framework? This is not obvious-especially in the case of nonlinear kinetics — and may be limited, at present, to the biological data available. What are the effects of discretizing both space and time? How do such models compare with those in which both are left as continuum variables [135]? Can efficient numerical code be developed to simulate large numbers of realizations of stochastic systems with multiple, interacting species? This is becoming increasingly important as our knowledge of biological systems at the cellular/molecular levels increases rapidly. How may different temporal and spatial scales be separated using asymptotic techniques? How can cellular level, stochastic dynamics (including phenomena such as feedback loops, amplifiers, switches) be incorporated into higher level models? Phenomena such as delays in gene transcription and translation and growth are well documented biologically, but how do they interact and how does stochasticity play a role?

\subsection{Domain geometry and heterogeneity in the developmental setting}

In the above we have seen that a major challenge in modelling is the development of methods for dealing with the numerous, complex biological interactions taking place during development. Revolutions in the coupling of molecular biology and biological imaging entail that far greater knowledge is becoming available and will continue to become available, concerning the detailed geometry of development. For example, spatial and temporal gene expression 


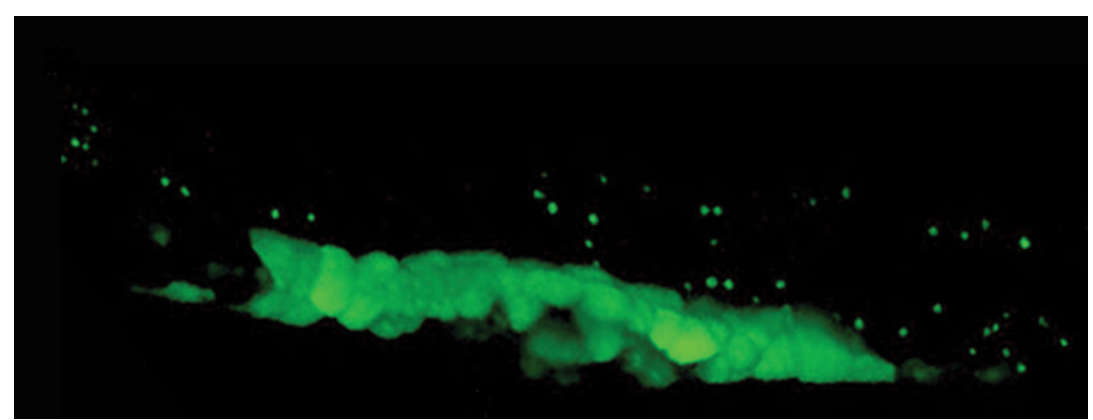

Figure 14. The developing uterus in C. Elegans; detailed information on expression of gene Y47G6a.7. Reproduced from [136].

profiles are available for approximately $10 \%$ of the genes within Caenorhabditis elegans [136]; similarly detailed geometrical information of domain geometries is becoming available, as illustrated in figure 14. In contrast, most pattern formation models consider a very simple domain, with transport modelled by Fickian diffusion. Consideration of events taking place on the cellular level immediately reveals that the basis of assumptions such as Fickian diffusion of signalling molecules and cells need to be investigated in detail. This is considered below, making an assumption of geometric periodicity at the cellular level, followed by an investigation of the effects of the macroscale geometry on important patterning properties such as stability and robustness.

3.3.1. Microgeometry: the cellular microenvironment. Consider figure 15. A priori, it is not clear that one can model transport within a complex extra-cellular domain using simple, Fickian diffusion, nor whether one can incorporate detailed intercellular dynamics in a meanfield sense within pattern formation models. Below we invoke homogenization theory [137] to show this is indeed possible. The technique, at the simplest level, is to perform a multiple scales perturbation analysis; the small parameter, $\epsilon$, is the ratio of the mesoscopic length scale, over which the system exhibits periodicity, to the cellular length scale, over which there is extensive heterogeneity. Below we will assume periodicity on the mesoscopic scale. It would also be appropriate to consider media which are random on the length scale of a few cells, though statistically homogeneous on the mesocopic length scale. We note that such techniques are common in other fields, such as the modelling of dislocations and shocks in physics [138] and theoretical electrophysiology [137,139], but have yet to become standard in biological self-organization modelling.

To begin, consider the microscale equations for two extracellular species $U_{1}, U_{2}$, whose concentrations in the extracellular space are denoted by $u_{1}$ and $u_{2}$, respectively. As we are considering the cellular level geometry, we also need to consider the dynamics on this scale. We assume there is a surface density of free receptors, denoted $r_{0}$, which will bind to either species, forming bound receptors, $r_{1}$ or $r_{2}$, according to the binding molecule. As an example one could assume that the binding of $U_{1}$ induces an intracellular signal which leads to the production of both species $U_{1}, U_{2}$ subject to inhibition due to the binding of $U_{2}$. This illustrative model will show, inter-alia, how receptor competition at the cell surface can be incorporated into macroscopic equations.

The equations for the above framework are given by

$$
\frac{\partial u_{\alpha}}{\partial t}=\nabla \cdot\left(D_{\alpha} \nabla u_{\alpha}\right)
$$




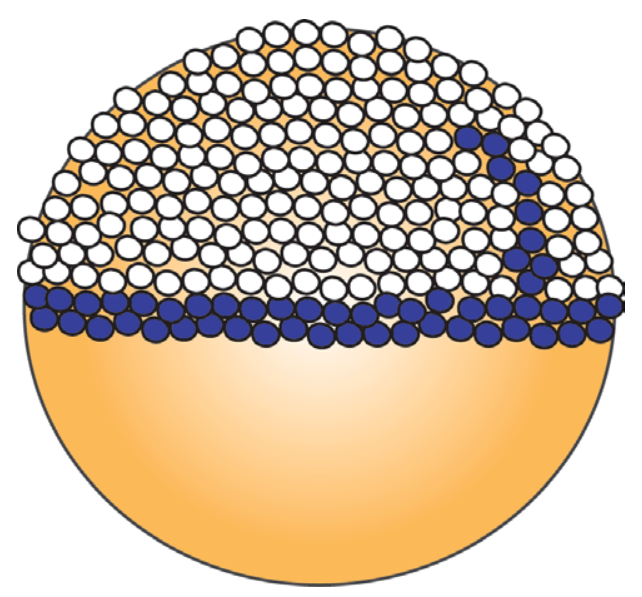

Figure 15. A cartoon of the tortuous microscale scale geometry of cells (white and shaded) plus the extracellular space in the context of zebrafish gastrula at $50 \%$ epiboly. In the figure, shading represents cells which express the mesodermal marker no tail (ntl) in wild type. Studies of Nodal and Lefty in the lead up to this stage of zebrafish development indicate that these proteins form a Turing pair controlling the balance of mesodermal versus ectodermal cells [6]. Figure reproduced with slight modifications from [6].

for $\alpha \in\{1,2\}$, within the complicated extracellular domain, denoted $\Omega_{e}$. Note that we do not have the summation convention for the repeated Greek subscripts. On the cell surfaces, that is on $\partial \Omega_{e}$, we also have interaction of the chemical species and receptors:

$$
\begin{aligned}
& D_{\alpha} \boldsymbol{n} \cdot \nabla u_{\alpha}=\epsilon A_{\alpha}\left(u_{1}, u_{2}, r_{0}, r_{1}, r_{2}\right), \\
& \frac{\partial r_{\beta}}{\partial t}=B_{\beta}\left(u_{1}, u_{2}, r_{0}, r_{1}, r_{2}\right),
\end{aligned}
$$

where $\beta \in\{0,1,2\}$ and $\boldsymbol{n}$ is the normal pointing out of the extra-cellular domain. We keep these cell surface interactions in a general setting during the model formulation stage to emphasize the universal application of this technique.

In practice, the scaling with $\epsilon$ for the surface fluxes in equation (78) emerges during non-dimensionalization, as illustrated in cardiac electrophysiological modelling [139, 140]. However, one can anticipate the above scalings; as $\epsilon$ tends to zero, with the macroscopic length scale fixed, and the cellular length scale decreasing, the surface area per cell scales with $\epsilon^{2}$. The number of cells in a fixed macroscopic volume scales with $1 / \epsilon^{3}$ and thus for a fixed surface density of receptors, the number of receptors in a unit macroscopic volume would scale as $1 / \epsilon$ as $\epsilon \rightarrow 0$. To avoid a divergence, or an absence of, receptors in the asymptotic limit, one must have the receptor surface-density scaling with $\epsilon$. For typical receptor dynamics, the functions $A_{\alpha}$ and $B_{\beta}$ will be homogeneous degree one in receptor density, giving the above on a simple rescaling of $r_{0}, r_{1}$ and $r_{2}$ to $\epsilon$-independent densities. Of course, the biophysical parameters used for non-dimensionalization, and thus $\epsilon$, are fixed; we simply find an approximation to the modelling equations which is accurate under the well-justified assumption that $\epsilon \ll 1$. $^{3}$

To implement the multiple scales analysis, let $\boldsymbol{x}$ denote the spatial coordinates which vary on the macroscopic length scale; similarly, $\boldsymbol{y}=\boldsymbol{x} / \epsilon$ are the spatial coordinates which vary

\footnotetext{
3 The biophysical parameters may in fact indicate that an excellent approximation is achieved with the absence of receptors or for a large receptor reservoir, which would yield different scalings to those above; we implicitly assume that no such simplifications occur in the model formulation.
} 
on the cellular scale. Thus, a function, say $f(\boldsymbol{x}, \boldsymbol{y})$, which depends on both macroscopic and cellular length scales has a gradient with respect to the macroscopic length scale of

$$
[\nabla f]_{i}=\frac{\partial f}{\partial x_{i}}+\frac{1}{\epsilon} \frac{\partial f}{\partial y_{i}},
$$

where the Latin index, $i \in\{1,2,3\}$ denotes the $i$ th spatial coordinate. One can readily deduce analogous expressions for other differential operators. In addition, assuming the cellular level fluctuations perturb the mean field, we have

$$
\begin{aligned}
& u_{\alpha}(\boldsymbol{x}, \boldsymbol{y}, t)=u_{\alpha}^{0}(\boldsymbol{x}, t)+\epsilon u_{\alpha}^{1}(\boldsymbol{x}, \boldsymbol{y}, t)+\epsilon^{2} u_{\alpha}^{2}(\boldsymbol{x}, \boldsymbol{y}, t)+\cdots, \quad \alpha \in\{1,2\}, \\
& r_{\beta}(\boldsymbol{x}, \boldsymbol{y}, t)=r_{\beta}^{0}(\boldsymbol{x}, t)+\epsilon r_{\beta}^{1}(\boldsymbol{x}, \boldsymbol{y}, t)+\epsilon^{2} r_{\beta}^{2}(\boldsymbol{x}, \boldsymbol{y}, t)+\cdots, \quad \beta \in\{0,1,2\} .
\end{aligned}
$$

Substituting the expansions of the differential operators and variables into equations (76) and (78), we have at order $\epsilon^{-1}$

$$
\begin{aligned}
& 0=\frac{\partial^{2} u_{\alpha}^{1}}{\partial y_{i} \partial y_{i}}, \\
& 0=D_{\alpha} n_{i}\left[\frac{\partial u_{\alpha}^{0}}{\partial x_{i}}+\frac{\partial u_{\alpha}^{1}}{\partial y_{i}}\right],
\end{aligned}
$$

where summation convention is implicit for repeated Latin indices. Similarly, at order $\epsilon^{0}$ we have

$$
\begin{aligned}
\frac{\partial u_{\alpha}^{0}}{\partial t} & =D_{\alpha}\left[\frac{\partial^{2} u_{\alpha}^{0}}{\partial x_{i} \partial x_{i}}+2 \frac{\partial^{2} u_{\alpha}^{1}}{\partial x_{i} \partial y_{i}}+\frac{\partial^{2} u_{\alpha}^{2}}{\partial y_{i} \partial y_{i}}\right], \\
& =D_{\alpha}\left\{\frac{\partial}{\partial x_{i}}\left[\frac{\partial u_{\alpha}^{0}}{\partial x_{i}}+\frac{\partial u_{\alpha}^{1}}{\partial y_{i}}\right]+\frac{\partial}{\partial y_{i}}\left[\frac{\partial u_{\alpha}^{1}}{\partial x_{i}}+\frac{\partial u_{\alpha}^{2}}{\partial y_{i}}\right]\right\},
\end{aligned}
$$

and the surface relations

$$
\begin{aligned}
& D_{\alpha} n_{i}\left[\frac{\partial u_{\alpha}^{1}}{\partial x_{i}}+\frac{\partial u_{\alpha}^{2}}{\partial y_{i}}\right]=A_{\alpha}\left(u_{1}^{0}, u_{2}^{0}, r_{0}^{0}, r_{1}^{0}, r_{2}^{0}\right), \\
& \frac{\partial r_{\beta}^{0}}{\partial t}=B_{\beta}\left(u_{1}^{0}, u_{2}^{0}, r_{0}^{0}, r_{1}^{0}, r_{2}^{0}\right) .
\end{aligned}
$$

We impose equation (82) for $u_{\alpha}^{1}$ within the extracellular subspace of the smallest repeating subunit of the periodic domain, which is denoted by $\Omega_{p}$; see figure 16 and its caption for further details of the geometry. The solution is of the form

$$
u_{\alpha}^{1}(\boldsymbol{x}, \boldsymbol{y}, t)=w_{\alpha i}(\boldsymbol{y}) \frac{\partial u_{\alpha}^{0}(\boldsymbol{x}, t)}{\partial x_{i}}+v_{\alpha}(\boldsymbol{x}, t),
$$

where $u_{\alpha}^{0}, v_{\alpha}$ are currently unknown and the $w_{\alpha i}$ satisfy

$$
\begin{aligned}
& \frac{\partial^{2} w_{\alpha i}}{\partial y_{j} \partial y_{j}}=0, \quad \boldsymbol{y} \in \Omega_{p}, \\
& n_{j}\left[\frac{\partial w_{\alpha i}}{\partial y_{j}}+\delta_{i j}\right]=0, \quad \boldsymbol{y} \in \partial \Omega_{p} \cap \partial \Omega_{e}, \\
& w_{\alpha i} \text { periodic on } \boldsymbol{y} \in \partial \Omega_{p} \backslash\left(\partial \Omega_{e} \cap \Omega_{p}\right) .
\end{aligned}
$$

Further, without loss of generality, one can take $v_{\alpha} \equiv 0$ by inspection of (80). One can also extend $w_{\alpha 1}, w_{\alpha 2}$ to the whole domain by periodicity ${ }^{4}$. We also have that equation (88) implies

\footnotetext{
4 To construct a twice differentiable solution in this manner will require additional constraints to those stated thus far; it would be sufficient for $\Omega_{p}$ to exhibit reflection symmetries in planes perpendicular to its principal axes and containing its centroid.
} 


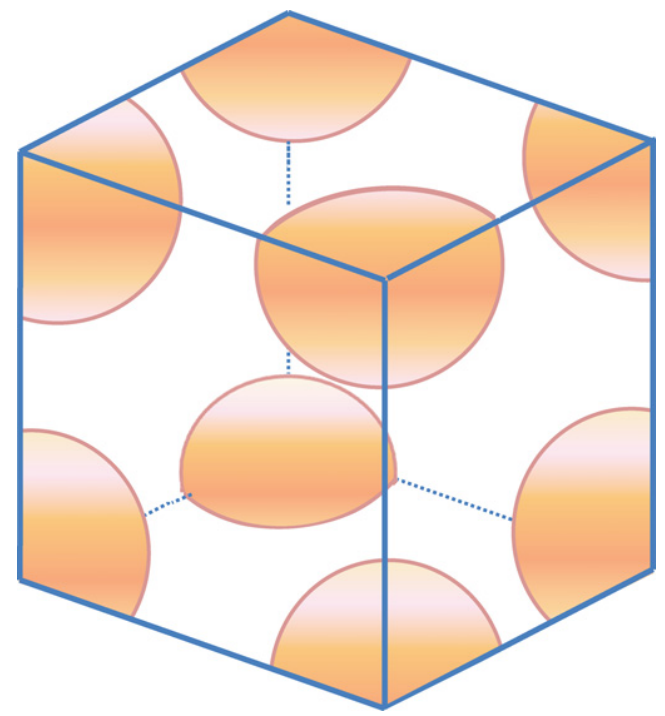

Figure 16. A description of the geometry used in the multiple scales analysis. For illustrative purposes, the smallest self-repeating unit is depicted as a cuboid, whose edges are shown in blue. In general this contains several cells, or parts of cells, as illustrated in orange; it is also, for definiteness, taken to contain the point $\boldsymbol{x}$. The domain $\Omega_{p}$ considered in the text is the extracellular space contained within the depicted cuboid. The boundary $\partial \Omega_{p}$ is equal to the union of $\partial \Omega_{p} \cap \partial \Omega_{e}$ and $\partial \Omega_{p} \backslash\left(\partial \Omega_{e} \cap \Omega_{p}\right)$, where $\Omega_{e}$ denotes the extracellular space; in particular, neither $\partial \Omega_{p} \cap \partial \Omega_{e}$ nor $\partial \Omega_{p} \backslash\left(\partial \Omega_{e} \cap \Omega_{p}\right)$ is empty in general.

$w_{\alpha 1}=w_{\alpha 2}$, at least to within a constant; the fact that $w_{\alpha 1}, w_{\alpha 2}$ are only uniquely defined up to a constant will not be important below.

We are now in a position to homogenize. Note that in the multiple scales asymptotic approximation one seeks solutions treating the spatial variables $\boldsymbol{x}$ and $\boldsymbol{y}$ as independent once any differential operators have been expanded via equation (79) and its analogues [141]. Thus, the multiple scales approach allows us to integrate equation (85) with respect to the cellular scale variable $\boldsymbol{y}$ over $\Omega_{p}$. On dividing through by the volume of $\Omega_{p}$, and using (85), (87) and (88), to eliminate $u_{\alpha}^{1}, u_{\alpha}^{2}$ we obtain

$$
\frac{\partial u_{\alpha}^{0}}{\partial t}=D_{\alpha}\left\{\delta_{i j}+\left[\frac{\int_{\Omega_{p}} \frac{\partial w_{\alpha i}}{\partial y_{j}} \mathrm{~d} V_{y}}{\int_{\Omega_{p}} \mathrm{~d} V_{y}}\right]\right\} \frac{\partial^{2} u_{\alpha}^{0}}{\partial x_{i} \partial x_{j}}+\lambda A_{\alpha}\left(u_{1}^{0}, u_{2}^{0}, r_{0}^{0}, r_{1}^{0}, r_{2}^{0}\right) .
$$

The parameter $\lambda$ is the area of $\partial \Omega_{e} \cap \partial \Omega_{p}$ divided by the volume of $\Omega_{p}$, which is equivalent to the surface area-volume ratio of the extracellular domain. Note that our homogenized equation depends only on derivatives of $w_{\alpha 1}, w_{\alpha 2}$ and is thus unique despite the fact that $w_{\alpha 1}, w_{\alpha 2}$ are unique only up to a constant. We also have the zero order receptor equations

$$
\frac{\partial r_{\beta}^{0}}{\partial t}=B_{\beta}\left(u_{1}^{0}, u_{2}^{0}, r_{0}^{0}, r_{1}^{0}, r_{2}^{0}\right) \text {, }
$$

which depend on $\boldsymbol{x}$ and $t$, but not $\boldsymbol{y}$, as required for consistency. Thus equations (88)-(90) dictate how the cellular level features, such as the microgeometry and receptor dynamics, are incorporated into macroscopic models, accurate to leading order in $\epsilon$.

In particular, note that microscale anisotropy will manifest in a more complicated macroscale diffusion operator and receptor dynamics are inherited essentially unchanged 
at the larger scale. These dynamics could be based upon simply the law of mass action or include, for example, simple representations of a gene expression time delay. A similar procedure, explicitly taking into account the cell interior, could readily incorporate a coupling of the receptors with signal transduction pathways and intracellular dynamics, allowing the systematic construction of multiscale models for the interaction of subcellular and supercellular phenomena.

An important constraint for the application of this procedure is that a mean-field approximation is appropriate. For example, it would not be immediately applicable to the fine-grained patterning associated with delta-notch lateral inhibition as a discrete cellularscale model is required [1]. Such exceptions aside, this homogenization procedure provides a framework for the construction of a single multiscale model for variations on the macroscale which incorporates (i) cellular level geometrical data from imaging, (ii) the ever increasing details becoming available at the networks and pathways level and (iii) cell surface kinetic data derived from FRAP studies [110].

This also serendipitously highlights a broad open challenge. Can the extensive literature on homogenization procedures (e.g. [138]) be adapted to produce accurate, simpler, macroscale models of biological self-organization in the presence of discrete modelling frameworks or stochasticity, such as low gene expression copy number fluctuations? Complications arise in such scenarios as the smoothness and determinism of the modelling variables on the cellular scale would generally be absent.

3.3.2. Macrogeometry. At the macroscale level in development, one must also consider the fact that the domain is growing. Typically the domain growth rate is much slower than other processes, allowing an effective and simplifying decoupling. Even when the domain growth drives the system through a patterning bifurcation it is typically quasi-steady either side of the bifurcation point and thus the homogenization analysis of section 3.3.1 is expected to be legitimate. This shows that while domain growth cannot simply be ignored, it can be represented by a slow time evolution of model parameters independent of microlevel complexities.

The consequences of domain growth upon self-organization frameworks such as the morphogen or Turing models have been investigated extensively using PDEs and the conservation approach [142-145]. Starting, once again, from the basic format assumed in equation (1) we have

$$
\frac{\partial}{\partial t} \int_{\Omega_{a}} \boldsymbol{u}(\boldsymbol{x}, t) \mathrm{d} \boldsymbol{x}=-\int_{\partial \Omega_{a}} \boldsymbol{J} \cdot \mathrm{d} \boldsymbol{s}+\int_{\Omega_{a}} \boldsymbol{f}(\boldsymbol{u}, \boldsymbol{x}, t) \mathrm{d} \boldsymbol{x},
$$

with the same notation. Supposing that $\Omega_{a}(t)$ is an arbitrary volume in a time-varying domain $\Omega(t)$, we draw on the Reynolds transport theorem [146] to express the left-hand side as

$$
\frac{\partial}{\partial t} \int_{\Omega_{a}} \boldsymbol{u}(\boldsymbol{x}, t) \mathrm{d} \boldsymbol{x}=\int_{\Omega_{a}}\left[\frac{\partial \boldsymbol{u}}{\partial t}+\nabla \cdot(\boldsymbol{a u})\right] \mathrm{d} \boldsymbol{x},
$$

which gives

$$
\frac{\partial \boldsymbol{u}}{\partial t}+\nabla \cdot(\boldsymbol{a u})=\nabla \cdot \boldsymbol{J}+\boldsymbol{f}(\boldsymbol{u}, \boldsymbol{x}, t)
$$

where $\boldsymbol{a}$ is the flow due to domain growth. On incorporating domain growth, two new terms emerge in the model: the first, $\boldsymbol{a} \cdot \nabla \boldsymbol{u}$, is an advection term which corresponds to flow due to local growth and the second, $\boldsymbol{u} \nabla \cdot \boldsymbol{a}$, to the dilution effects of local volume change.

A Lagrangian description may be applied to the problem:

$$
\boldsymbol{x}=\boldsymbol{\Gamma}(\boldsymbol{X}, t), \quad \boldsymbol{x} \in \boldsymbol{\Omega}(t),
$$


where $\boldsymbol{X}$ is the initial position of an element moving in the flow, $\boldsymbol{a}$, so that $\boldsymbol{\Gamma}(\boldsymbol{X}, 0)=\boldsymbol{X}$. The local flow can then be specified as

$$
\boldsymbol{a}(\boldsymbol{X}, t)=\frac{\partial \boldsymbol{x}}{\partial t}=\frac{\partial \boldsymbol{\Gamma}}{\partial t}
$$

where the mechanical properties of the tissue have been ignored and growth only assumed to occur due to local volumetric increase [147].

Crampin and co-workers considered pattern formation in Turing-type reaction-diffusion models with domain growth. Beginning with slow isotropic growth, they demonstrate pattern formation under different types of growth function and show that domain growth may be a mechanism for increased robustness of pattern formation [147]. In a further work, they generalize their results to non-uniformly growing domains, showing that weak spatial heterogeneity does not alter pattern selection from the uniform case, but that sufficient nonuniformity can give rise to new pattern sequences [145].

Crampin and co-workers further extended their study to consider Turing instabilities when growth was coupled to morphogen concentration [145]. However, they did not consider any specific biological applications. Baker and co-workers [142] consider simple morphogen models, in which local morphogen concentrations were assumed to control cell proliferation (and hence domain growth) in a manner similar to that shown to control cell proliferation in the Drosophila wing disc [148]: growth could either arise if the gradient or the absolute levels of Dpp concentration were above certain thresholds. In certain limits, they were able to derive analytical expressions describing domain growth over time.

Further to this work, Neville et al [149] considered the pattern forming potential of the Schnakenberg model on growing domains, comparing isotropic, exponential domain growth with scenarios with biochemically controlled growth. They demonstrated, via numerical simulations and weakly nonlinear analysis, that despite a complex interplay between the effects of chemicals on the domain and the influence of the domain on the patterns formed, domain growth increases the tendency of the uniform steady state to form patterns.

However, all such models consider very simple geometries, and thus an interesting development will be pattern formation on differential manifolds incorporating, for example, the Laplace Beltrami operator for diffusive processes [150,151]. In addition, numerous open problems emerge when considering how to cross the scale between movement on cellular scales and global domain growth. One example is the generalization of the above homogenization procedures when microscale periodicity is absent. Another example is revealed on noting that, in contrast to individual cell motion during mesenchymal morphogenesis, numerous migratory movements during development occur via cell sheets. Self-organization models taking into account such coordinated movement are in their infancy and determining how to incorporate detailed cell sheet movements within domain growth via mean-field equations represents a major challenge.

We conclude this section by noting that pattern formation under the influence of domain growth, and the resultant increased levels of robustness, is an example of sequential induction [1], whereby a complicated pattern is reliably formed by a sequence of patterning forming events, as, indeed, suggested by Turing. Sequential induction is considered to be a common mechanism throughout development, with differential gene expression acting temporally as well as spatially [1]. Consequently, it is clear that, more generally, the slow evolution of parameters within biological models of pattern formation should also be investigated in detail to see how temporal regulation can assist in invoking and maintaining pattern. 


\section{Summary}

We began this work by outlining some of the areas in which PDEs have already been used to model developmental systems, sketching a derivation of the PDE formulation using the principle of conservation and discussing the suitability of PDEs for modelling biological systems. In section 2 we gave an overview of some classical paradigm models, showing some of the mathematical techniques commonly used in their analyses and presenting results of numerical simulations. In section 3 we discussed some of the most pertinent open problems in the area, including the integration of large amounts of molecular data and noise.

The future of PDE modelling in developmental biology now lies in researchers embracing challenges such as those summarized here. We must be respectful of the fact that simple models do have many advantages — not least their amenability to analytical exploration — whilst being aware of the enormous complexity of biological systems now becoming apparent with the recent revolution in molecular biology.

\section{Acknowledgments}

REB would like to thank Microsoft Research for a European Postdoctoral Fellowship, Research Councils, UK, for an RCUK Fellowship in Mathematical Biology and St Hugh's College, Oxford for a Junior Research Fellowship. PKM was partly supported by a Royal SocietyWolfson Merit Award.

\section{References}

[1] Alberts B, Johnson A, Lewis J, Raff M, Roberts K and Walter P 2002 Molecular Biology of the Cell 4th edn (London: Garland Science, Taylor and Francis)

[2] Hamada H, Meno C, Watanabe D and Saijoh Y 2002 Establishment of vertebrate left-right asymmetry Nat. Rev. Genet. 3 103-13

[3] Raya A and Izpisúa-Belmonte J C 2006 Left-right asymmetry in the vertebrate embryo: from early information to higher-level integration Nat. Rev. Genet. 7 283-93

[4] Smith D J, Blake J R and Gaffney E A 2008 Fluid mechanics of nodal flow due to embryonic primary cilia $J$. R. Soc. Interface 5 567-73

[5] Tabin C J 2006 The key to left-right asymmetry Cell 127 27-32

[6] Solnica-Krezel L 2003 Vertebrate development: taming the nodal waves Curr. Biol. 13 R7-9

[7] De Celis J F 2003 Pattern formation in the Drosophila wing: the development of the veins BioEssays 25 443-51

[8] Sick S, Reinker S, Timmer J and Schlake T 2006 WNT and DKK determine hair follicle spacing through a reaction-diffusion mechanism Science 314 1447-50

[9] Affolter M, Bellusci S, Itoh N, Shilo B, Thiery J P and Werb Z 2003 Tube or not tube: remodeling epithelial tissues by branching morphogenesis Dev. Cell 4 11-8

[10] Simpson M J, Zhang D C, Mariani M, Landman K A and Newgreen D F 2007 Cell proliferation drives neural crest cell invasion of the intestine Dev. Biol. 302 553-68

[11] Yang X, Dormann D, Munsterberg A E and Weijer C J 2002 Cell movement patterns during gastrulation in the chick are controlled by positive and negative chemotaxis mediated by FGF4 and FGF8 Dev. Cell 3 425-37

[12] Gossler A and Hrabě de Angelis M 1998 Somitogenesis Curr. Top. Dev. Biol. 38 225-87

[13] Baker R E, Schnell S and Maini P K 2008 Mathematical models for somite formation Current Topics in Developmental Biology: Multiscale Modeling of Developmental Systems (New York: Academic) p 183203

[14] Murray J D, Oster G F and Harris A K 1983 A mechanical model for mesenchymal morphogenesis J. Math. Biol. 17 125-29

[15] Oster G F, Murray J D, Harris A K 1983 Mechanical aspects of mesenchymcal morphogenesis J. Embryol. Exp. Morphol. 78 83-125

[16] Murray J D, Maini P K, Tranquillo R T 1988 Mechanochemical models for generating biological pattern and form in development Phys. Rep. 171 59-84

[17] Newman S A 2007 The turing mechanism in vertebrate limb patterning Nat. Rev. Mol. Cell. Biol. 8 
[18] Bard J B L 1977 A unity underlying the different zebra striping patterns J. Zool. 183 527-39

[19] Bard J B L 1981 A model for generating aspects of zebra and other mammalian coat patterns J. Theor. Biol. $93363-85$

[20] Murray J D 1981 A pre-pattern formation mechanism for animal coat markings J. Theor. Biol. 88 161-99

[21] Jung H S et al 1998 Local inhibitory action of BMPs and their relationships with activators in feather formation: implications for periodic patterning Dev. Biol. 196 11-23

[22] Lin C M, Jiang Y J, Baker R E, Maini P K, Widelitz R B and Chuong C M 2008 Spots and stripes: pleomorphic patterning of stem cells via p-ERK-dependent cell-chemotaxis shown by feather morphogenesis and mathematical simulation, submitted

[23] Collier J R, Monk N A M, Maini P K and Lewis J H 1996 Pattern formation by lateral inhibition with feedback: a mathematical model of delta-notch intercellular signalling J. Theor. Biol. 183 429-46

[24] Armstrong N J, Painter K J and Sherratt J A 2006 A continuum approach to modelling cell-cell adhesion $J$. Theor. Biol. 243 98-113

[25] Foty R A and Steinberg M S 2005 The differential adhesion hypothesis: a direct evaluation Dev. Biol. $278255-63$

[26] Kondo S and Asai R 1995 A reaction-diffusion wave on the skin of the marine angelfish Pomacanthus Nature 376 765-68

[27] Suzuki N, Hirata M and Kondo S 2003 Traveling stripes on the skin of a mutant mouse Proc. Natl Acad. Sci. USA $1009680-5$

[28] Budrene E O and Berg H C 1995 Dynamics of formation of symmetrical patterns by chemotactic bacteria Nature 376 49-53

[29] Eisenbach J W M with contributions from Lengeler J W et al 2004 Chemotaxis (London: Imperial College Press)

[30] Nagano S 2000 Modeling the model organism Dictyostelium discoideum Dev. Growth Differ. 42 541-50

[31] Murray J D 2003 Mathematical Biology: I. An Introduction vol I 3rd edn (Berlin: Springer)

[32] Murray J D 2003 Mathematical Biology: II. Spatial Models and Biochemical Applications vol II 3rd edn (Berlin: Springer)

[33] Sekimura T, Zhu M, Cook J, Maini P K and Murray J D 1999 Pattern formation of scale cells in lepidoptera by differential origin-dependent cell adhesion Bull. Math. Biol. 61 807-27

[34] Cohen D S and Murray J D 1981 A generalized diffusion model for growth and dispersal in a population $J$. Math. Biol. 12 237-49

[35] Othmer H G, Dunbar S R and Alt W 1988 Models of dispersal in biological systems J. Math. Biol. 26 263-98

[36] Ockendon J R, Howison S D, Lacey A A and Movchan A B 2003 Applied Partial Differential Equations 2nd edn (Oxford: Oxford University Press)

[37] Othmer H G and Stevens A 1997 Aggregation, blowup, and collapse: The ABC's of taxis in reinforced random walks SIAM J. Appl. Math. 57 1044-81

[38] Hillen T and Othmer H G 2000 The diffusion limit of transport equations derived from velocity-jump processes SIAM J. Appl. Math. 61 751-75

[39] Painter K J, Maini P K and Othmer H G 2000 Development and applications of a model for cellular response to multiple chemotactic cues J. Math. Biol. 41 285-314

[40] Cross M C and Hohenberg P C 1993 Pattern formation outside of equilibrium Rev. Mod. Phys. 65851

[41] Britton N F 1987 Reaction-Diffusion Equations and their Applications to Biology (New York: Academic)

[42] Fife P C 1979 Mathematical Aspects of Reacting and Diffusing Systems (Berlin: Springer)

[43] Grindrod P 1996 Patterns And Waves 2nd edn (Oxford: Oxford University Press)

[44] Hoyle R 2006 Pattern Formation: An Introduction to Methods (Oxford: Cambridge University Press)

[45] Wolpert L, Beddington R, Jessell T, Lawrence P, Meyerowitz E and Smith J 2006 Principles of Development 3rd edn (Oxford: Oxford University Press)

[46] Dessaud E et al 2007 Interpretation of the sonic hedgehog morphogen gradient by a temporal adaptation mechanism Nature 450 717-20

[47] Pagès F and Stephen K 2000 Morphogen gradients: a question of time or concentration? Trends Genet. 16 40-4

[48] Tickle C 1999 Morphogen gradients in vertebrate limb development Cell Dev. Biol. 10 345-51

[49] Tickle C 2006 Making digit patterns in the vertebrate limb Nat. Rev. Mol. Cell. Biol. 7 45-53

[50] Towers M, Mahood R, Yin Y and Tickle C 2008 Integration of growth and specification in chick wing digitpatterning Nature 452 882-6

[51] Entchev E V, Schwabedissen A and González-Gaitán M M 2000 Gradient formation of the TGF- $\beta$ homolog Dpp Cell 103 981-91

[52] Teleman A A and Cohen S M 2000 Dpp gradient formation in the Drosophila wing imaginal disc Cell $103971-80$ 
[53] Diez del Corral R, Olivera-Martinez I, Goriely A, Gale E, Maden M and Storey K 2003 Opposing FGF and retinoid pathways control ventral neural pattern, neuronal differentiation and segmentation during body axis extension Neuron 40 65-79

[54] Diez del Corral R and Storey K G 2004 Opposing FGF and retinoid pathways: a signalling switch that controls differentiation and patterning onset in the extending vertebrate body axis BioEssays 26 857-69

[55] Eldar A, Dorfman R, Weiss D, Ashe H, Shilo B Z and Barkai N 2002 Robustness of the BMP morphogen gradient in Drosophila embryonic patterning Nature $419304-8$

[56] Turing A M 1952 The chemical basis of morphogenesis R. Soc. Lond. Phil. Trans. B 237 37-72

[57] Satnoianu R A, Menzinger M and Maini P K 2000 Turing instabilities in general systems J. Math. Biol. 41 493-512

[58] Meinhardt H 1982 Models of Biological Pattern Formation (London: Academic)

[59] Gierer A and Meinhardt H 1972 A theory of biological pattern formation Kybernetik 12 30-9

[60] Baker R E, Schnell S and Maini P K 2008 Waves and patterning in developmental biology: vertebrate segmentation and feather bud formation as case studies Int. J. Dev. Biol. at press

[61] Shampine L F 2007 Accurate numerical derivatives in MATLAB ACM Trans. Math. Softw. 3326

[62] Miura T, Shiota K, Morriss-Kay G and Maini P K 2006 Mixed-mode pattern in Doublefoot mutant mouse limb - Turing reaction-diffusion model on a growing domain during limb development J. Theor. Biol. $240562-73$

[63] Richter P H, Rehmus P and Ross J 1981 Control and dissipation in oscillatory chemical engines Prog. Theor. Phys. 66 385-405

[64] Eldar A, Shilo B Z and Barkai N 2004 Elucidating mechanisms underlying robustness of morphogen gradients Curr. Op. Genet. Dev. 14 435-9

[65] Liu L, Ratner B D, Sage E H and Jiang S 2007 Endothelial cell migration on surface-density gradients of fibronectin, VEGF, or both proteins Langmuir 23 11168-73

[66] Smith J T, Tomfohr J K, Wells M C, Beebe T P, Kepler T B and Reichert W M 2004 Measurement of cell migration on surface-bound fibronectin gradients Langmuir 20 8279-86

[67] von der Hardt S, Bakkers J, Inbal A, Heisenberg C P and Hammerschmidt M 2007 The BMP gradient of the zebrafish gastrula guides migrating lateral cells by regulating cell-cell adhesion Curr. Biol. 17 475-87

[68] Gomez T M and Letourneau P C 1994 Filopodia initiate choices made by sensory neuron growth cones at laminin/fibronectin borders in vitro J. Neurosci. 14 5959-72

[69] Gray D S, Tien J and Chen C S 2003 Repositioning of cells by mechanotaxis on surfaces with micropatterned Young's modulus J. Biomed. Mater. Res. A 66 605-14

[70] Li S et al 2002 The role of the dynamics of focal adhesion kinase in the mechanotaxis of endothelial cells Proc. Natl Acad. Sci. USA 99 3546-51

[71] Adams C F and Paul A J 1999 Phototaxis and geotaxis of light-adapted zoeae of the golden king crab lithodes aequispinus (anomura: Lithodidae) in the laboratory J. Crustacean. Biol. 19 106-10

[72] McCaig C D, Rajnicek A M, Song B and Zhao M 2005 Controlling cell behavior electrically: current views and future potential Phys. Rev. 85 943-78

[73] Blakemore R P 1982 Magnetotactic bacteria Annu. Rev. Microbiol. 36 217-38

[74] Fenchel T and Finlay B J 1984 Geotaxis in the ciliated protozoon loxodes J. Exp. Biol. 110 17-33

[75] Jones M S, Le Baron L and Pedley T J 1994 Biflagellate gyrotaxis in a shear flow J. Fluid Mech. 281 137-58

[76] Schnell S, Painter K J, Maini P K and Othmer H G 2000 Spatiotemporal pattern formation in early development: a review of primitive streak formation and somitogenesis ed P K Maini and H G Othmer Mathematical Models for Biological Pattern Formation (IMA Volumes in Mathematics and its Applications vol 121) (New York: Springer) p 11-37

[77] Painter K J, Maini P K and Othmer H G 2000 A chemotactic model for the advance and retreat of the primitive streak in avian development Bull. Math. Biol. 62 501-25

[78] Painter K J, Maini P K and Othmer H G 1999 Stripe formation in juvenile Pomacanthus explained by a generalized Turing mechanism with chemotaxis Proc. Natl Acad. Sci. USA 96 5549-54

[79] Segel L A 1977 A theoretical study of receptor mechanisms in bacterial chemotaxis SIAM J. Appl. Math. 32 653-65

[80] Othmer H G and Hillen T 2002 The diffusion limit of transport equations II: chemotaxis equations SIAM J. Appl. Math. 62 1222-50

[81] Myerscough M R, Maini P K, Murray J D and Winters K H 1990 Two dimensional pattern formation in a chemotactic system Dynamics of Complex Interconnected Biological Systems (Boston, MA: Birkhauser) pp 65-83

[82] Myerscough M R, Maini P K and Painter K J 1998 Pattern formation in a generalised chemotactic model Bull. Math. Biol. 60 1-26 
[83] Eldar A, Rosin D, Shilo B Z and Barkai N 2003 Self-enhanced ligand degradation underlies robustness of morphogen gradients Dev. Cell. 5 635-46

[84] Asai R, Taguchi E, Kume Y, Saito M and Kondo S 1999 Zebrafish Leopard gene as a component of the putative reaction-diffusion system Mech. Dev. 89 87-92

[85] Akam M 1989 Making stripes inelegantly Nature 341 282-3

[86] Cruywagen G and Murray J 1992 On a tissue interaction model for skin pattern formation J. Nonlinear Sci. $2217-40$

[87] Cruywagen G C 1992 Tissue interaction and spatial pattern formation PhD Thesis (Oxford: Oxford University Press)

[88] Ward M J, McInerney D, Houston P, Gavaghan D J and Maini P K 2002 The dynamics and pinning of a spike for a reaction-diffusion system SIAM J. Appl. Math. 62 1297-328

[89] Cruywagen G C, Maini P K and Murray J D 1992 Sequential pattern formation in a model for skin morphogenesis IMA J. Math. Appl. Med. Biol. 9 227-48

[90] Cruywagen G C, Maini P K and Murray J D 1994 Bifurcating spatial patterns arising from travelling waves in a tissue interaction model Appl. Math. Lett. 7 3-66

[91] Cruywagen G C, Murray J D and Maini P K 2000 An envelope method for analyzing sequential pattern formation SIAM J. Appl. Math. $61213-31$

[92] Miura T and Shiota K 2000 Extracellular matrix environment influences chondrogenic pattern formation in limb bud micromass culture: experimental verification of theoretical models Anat. Rec. A 258 100-7

[93] Jiang T X, Jung H S, Widelitz R B and Chuong C M 1999 Self-organization of periodic patterns by dissociated feather mesenchymal cells and the regulation of size, number and spacing of primordia Development $\mathbf{1 2 6}$ 4997-5009

[94] Jiang T X et al 2004 Integument pattern formation involves genetic and epigenetic controls: feather arrays simulated by digital hormone models Int. J. Dev. Biol. 48 117-35

[95] Baker R E, Schnell S and Maini P K 2006 A clock and wavefront mechanism for somite formation Dev. Biol. 293 116-26

[96] Baker R E, Schnell S and Maini P K 2006 A mathematical investigation of a clock and wavefront model for somitogenesis J. Math. Biol. 52 458-82

[97] Chang C, Wu P, Baker R E, Maini P K, Alibardi L and Chuong CM 2008 The reptile scale paradigm: Evo-Devo, pattern formation and regeneration Int. J. Dev. Biol. at press

[98] Widelitz R B et al 2006 Distinct mechanisms underlie pattern formation in the skin and skin appendages Birth Defects Res C 78 280-91

[99] Monk N A M 2003 Oscillatory expression of Hes1, p53 and NF- $\kappa$ B driven by transcriptional time delays Curr. Biol. 13 1409-13

[100] Tennyson C N, Klamut H J and Worton R G 1995 The human dystrophin gene requires 16 hours to be transcribed and is cotranscriptionally spliced Nat. Genet. 9 184-90

[101] Gaffney E A and Monk N A M 2006 Gene expression time delays and turing pattern formation systems Bull. Math. Biol. 68 99-130

[102] von Dassow G, Meir E, Munro E M and Odell G M 2000 The segment polarity network is a robust developmental module Nature 406 188-92

[103] Shimizu T S, Aksenov S V and Bray D 2003 A spatially extended stochastic model of the bacterial chemotaxis signalling pathway J. Mol. Biol. 329 291-309

[104] Mincheva M and Roussel M R 2007 Graph-theoretic methods for the analysis of chemical and biochemical networks: I. Multistability and oscillations in ordinary differential equation models J. Math. Biol. 55 61-86

[105] Mincheva M and Roussel M R 2007 Graph-theoretic methods for the analysis of chemical and biochemical networks: II. Oscillations in networks with delays J. Math. Biol. 55 87-104

[106] Gaffney E A, Heath J K and Kwiatkowska M Z 2008 A mass action model of a fibroblast signalling pathway and its simplification Bull. Math. Biol. 70 2229-63

[107] Borisov N M, Markevich N I, Hoek J B and Kholodenko B N 2005 Signaling through receptors and scaffolds: independent interactions reduce combinatorial complexity Biophys. J. 89 951-66

[108] Borisov N M, Markevich N I, Hoek J B and Kholodenko B N 2006 Trading the micro-world of combinatorial complexity for the macro-world of protein interaction domains Biosystems 83 152-66

[109] Kicheva A et al 2007 Kinetics of morphogen gradient formation Science 315 521-5

[110] Dushek O and Coombs D 2008 Improving parameter estimation for cell surface FRAP data J. Biochem. Biophys. Methods 70 1224-31

[111] Strier D E and Dawson Ponce S 2007 Turing patterns inside cells PLoS ONE 2 e1053

[112] Rauch E M and Millonas M M 2004 The role of trans-membrane signal transduction in Turing-type cellular pattern formation $J$. Theor. Biol. 226 401-7 
[113] Basu S, Gerchman Y, Collins C H, Arnold F H and Weiss R 2005 A synthetic multicellular system for programmed pattern formation Nature $4341130-4$

[114] Raser J M and O'Shea E K 2004 Control of stochasticity in eukaryotic gene expression Science 304 1811-4

[115] Swain P S, Elowitz M B and Siggia E D 2002 Intrinsic and extrinsic contributions to stochasticity in gene expression Proc. Natl Acad. Sci. USA 99 12795-800

[116] Elowitz M B, Levine A J, Siggia E D and Swain P S 2002 Stochastic gene expression in a single cell Science $2971183-6$

[117] Kaern M, Elston T C, Blake W J and Collins J J 2005 Stochasticity in gene expression: from theories to phenotypes Nat. Rev. Genet. 6 451-64

[118] Erban R, Chapman S J and Maini P K 2007 A practical guide to stochastic simulations of reaction-diffusion processes. Available at http://arxiv.org/abs/0704.1908

[119] Briggs G E and Haldane J B S 1925 A note on the kinetics of enzyme action J. Biochem. 19339

[120] Gillespie D T 1977 Exact stochastic simulation of coupled chemical reactions J. Phys. Chem. 81 2340-61

[121] Tostevin F, ten Wolde P R and Howard M 2007 Fundamental limits to position determination by concentration gradients PLoS Comput. Biol. 3 e78

[122] Kruse K, Pantazis P, Bollenbach T, Jülicher F and González-Gaitán M 2004 Dpp gradient formation by dynamindependent endocytosis: receptor trafficking and the diffusion model Development $1314843-56$

[123] Bollenbach T, Kruse K, Pantazis P, González-Gaitán M and Jülicher F 2005 Robust formation of morphogen gradients Phys. Rev. Lett. 94 018103-1-018103-4

[124] Bollenbach T, Kruse K, Pantazis P, González-Gaitán M and Jülicher F 2007 Morphogen transport in epithelia Phys. Rev. E 75011901

[125] Baker R E and Maini P K 2007 Travelling gradients in interacting morphogen systems Math. Biosci. $20930-50$

[126] Dillon R, Gadgil C and Othmer H G 2003 Short- and long-range effects of sonic hedgehog in limb development Proc. Natl Acad. Sci. USA 100 10152-7

[127] Lander A D, Nie Q and Wan F Y M 2002 Do morphogen gradients arise by diffusion? Dev. Cell 2 785-96

[128] Lander A D, Nie Q and Wan F Y M 2005 Spatially distributed morphogen production and morphogen gradient formation Math. Biosci. Eng. 2 239-62

[129] Ashe H L and Briscoe J 2006 The interpretation of morphogen gradients Development 133 385-94

[130] Namba R, Pazdera T M, Cerrone R L and Minden J S 1997 Drosophila embryonic pattern repair: how embryos respond to bicoid dosage alteration Development 124 1393-403

[131] Dahmann C and Basler K 1999 Compartment boundaries: at the edge of development Trends Genet. 15 320-6

[132] Wijgerde M, McMahon J A, Rule M, and McMahon A P 2002 A direct requirement for Hedgehog signaling for normal specification of all ventral progenitor domains in the presumptive mammalian spinal cord Genes Dev. 16 2849-64

[133] Dubrulle J, McGrew M J and Pourquié O 2001 FGF signalling controls somite boundary position and regulates segmentation clock control of spatiotemporal Hox gene activation Cell 106 219-32

[134] Rao C V, Wolf D M and Arkin A P 2002 Control, exploitation and tolerance of intracellular noise Nature 420 231-7

[135] Andrews S S and Bray D 2004 Stochastic simulation of chemical reactions with spatial resolution and single molecule detail Phys. Biol. 1 137-51

[136] Hunt-Newbury R et al 2007 High-throughput in vivo analysis of gene expression in Caenorhabditis elegans PLoS Biol. 5 e237

[137] Keener J P and Sneyd J 1998 Mathematical Physiology (Interdisciplinary Applied Mathematics vol 8) 1st edn (New York: Springer)

[138] Weinan E, Engquist B, L Xiantao, Weiqing R and Vanden-Eijnden E 2007 Heterogeneous multiscale methods: a review Commun. Comput. Phys. 2 367-450

[139] Krassowska W and Neu J C 1994 Effective boundary conditions for syncytial tissues IEEE Trans. Biomed. Eng. 41 143-50

[140] Juhlin S P, Pormann J B and Pormann J B 1994 Dimensional comparison of the sawtooth pattern in transmembrane potential Comput. Cardiol. p 413-6

[141] Bender C M and Orszag S A 1999 Advanced Mathematical Methods for Scientists and Engineers: I. Asymptotic Methods and Perturbation Theory (New York: Springer)

[142] Baker R E and Maini P K 2007 A mechanism for morphogen-controlled domain growth J. Math. Biol. 54 597-622

[143] Crampin E J and Maini P K 2001 Modelling biological pattern formation: the role of domain growth Commun. Theor. Biol. 6 229-49 
[144] Crampin E J, Gaffney E A and Maini P K 2002 Mode-doubling and tripling in reaction-diffusion patterns on growing domains: a piecewise linear model J. Math. Biol. 44 107-28

[145] Crampin E J, Hackborn W W and Maini P K 2002 Pattern formation in reaction-diffusion models with nonuniform domain growth Bull. Math. Biol. 64 747-69

[146] Acheson D J 2005 Elementary Fluid Dynamics (Oxford Applied Mathematics and Computing Science Series) (Oxford: Clarendon)

[147] Crampin E J, Gaffney E A and Maini P K 1999 Reaction and diffusion on growing domains: scenarios for robust pattern formation Bull. Math. Biol. 61 1093-120

[148] Rogulja D and Irvine K D 2005 Regulation of cell proliferation by a morphogen gradient Cell 123 449-61

[149] Neville A, Matthews P and Byrne H 2006 Interactions between pattern formation and domain growth Bull. Math. Biol. 68 1975-2003

[150] Plaza R G, Sánchez-Garduno F, Padilla P, Barrio R A, Maini P K 2004 The effect of growth and curvature on pattern formation J. Dyn. Diff. Eqns 16 1093-121

[151] Chaplain M A, Ganesh M and Graham I G 2001 Spatio-temporal pattern formation on spherical surfaces: numerical simulation and application to solid tumour growth J. Math. Biol. 42 387-423 